\title{
Coupled Acoustic Mode Propagation Through Continental-Shelf Internal Solitary Waves
}

\author{
James C. Preisig, Member, IEEE, and Timothy F. Duda
}

\begin{abstract}
Three techniques are used to investigate mode coupling as acoustic energy passes through continental-shelf internal solitary waves (ISW's). Results from all techniques agree. The waves considered here are single downward undulations of a thermocline layer separating upper and lower well-mixed layers. Two techniques are numerical: parabolic equation (PE) solution and a sudden approximation joining range-invariant regions at sharp vertical interfaces. The third technique is an analytic derivation of ISW scale lengths separating adiabatic (at large scale) and coupled-mode propagation. Results show that energy is exchanged between modes as ISW's are traversed. The sharp interface solutions help explain this in terms of spatially confined coupling and modal phase interference. Three regimes are observed: 1) for short ISW's, coupling upon wave entrance is reversed upon exit, with no net coupling; 2) for ISW scales of 75-200 m, modal phase alteration averts the exit reversal, giving net coupling; transparent resonances yielding no net coupling are also observed in this regime; and 3 ) for long ISW's, adiabaticity is probable but not universal. Mode refraction analysis for nonparallel acoustic-ISW alignment suggests that these two-dimensional techniques remain valid for $0^{\circ}$ (parallel) to $65^{\circ}$ (oblique) incidence, with an accordant ISW stretching.
\end{abstract}

Index Terms - Coupled mode analysis, underwater acoustic propagation, underwater acoustics.

\section{INTRODUCTION}

$\mathbf{E}$ NERGETIC baroclinic tides and waves are a common feature of continental slope regions during stratified periods, having been observed in at least dozens of locales [1]. These often have characteristics consistent with internal solitary wave (ISW) or "soliton" mathematical solutions for stratified systems [2], [3] and usually appear as propagating pulse-like depressions of the thermocline. Since these waves can involve large vertical water displacements, up to roughly one-third the water depth, they cause a significant perturbation of the acoustic waveguide and are generally considered to be an important contributor to fluctuations of low-frequency acoustic signals. By low frequencies, we mean those welldescribed by less than 15 propagating modes, usually less than $1000 \mathrm{~Hz}$ on the continental shelf. The short scale (less than 200

Manuscript received July 16, 1996; revised February 7, 1997. This work was supported by the Office of Naval Research under Grant N00014-95-1-0029 and Grant N00014-95-1-0051. This is Woods Hole Oceanographic Institute Contribution 9294.

J. C. Preisig is with the Department of Applied Ocean Physics and Engineering, Woods Hole Oceanographic Institution, Woods Hole, MA 025431053 USA. He is also with the Department of Electrical and Computer Engineering, Northeastern University, Boston, MA, 02115 USA.

T. F. Duda is with the Department of Applied Ocean Physics and Engineering, Woods Hole Oceanographic Institution, Woods Hole, MA 02543-1053 USA.

Publisher Item Identifier S 0364-9059(97)03395-5. m) of the solitary waves and the large displacements suggest that adiabatic mode propagation criteria [4] may be violated and energy may scatter between modes. At least one published numerical propagation study has exhibited mode coupling as sound passes though thermocline fluctuations within a shallow waveguide [5].

Propagation modeling is undertaken here for the purpose of understanding which physical features of the waves are responsible for the coupling, what coupling mechanisms are important, and under what circumstances coupling may be expected. We use ISW shapes of the hyperbolic secant form, $\eta(x, t)=a \operatorname{sech}^{2}[(x-c t) / L]$, solutions of the Korteweg-de Vries equation, appropriate for the long-wave situation of solitary wave horizontal scale $L$ greater than the depth $(50 \mathrm{~m}$ throughout this paper). The sign of the solution is such that the waves are thermocline depressions if the stratification follows the general guidelines of an upper layer thinner than the deeper, denser layers. These ISW's resemble some observed in the New York Bight [6] where an acoustic experiment was completed in 1995 [7].

We use a three-layer system of sound speed, with a constantgradient transition layer between upper and lower mixed layers. This vertical structure is typical of many continentalshelf regions [6], [8]. We extend our investigation to solitary waves with amplitudes $(a)$ and horizontal scales not consistent with ISW solutions for our layering. The analysis of $(a, L)$ solitary-wave parameter pairs which do not satisfy the dispersion relation allows us to accommodate acoustic propagation through ISW's at other than normal incidence and gives us a more complete understanding of the relevant coupling mechanisms.

In studying the effects of ISW's on acoustic propagation, two numerical techniques are used. One is the wide-angle parabolic equation method (PE). The other is a suddenapproximation [9] coupled-mode propagation method which uses matrix manipulations, which we call the sharp-interface approximation (SIA). The PE is used to reliably compute acoustic fields in arbitrary ocean structures. The SIA, which has well-understood mode-coupling characteristics, can then be used to examine the important factors controlling mode coupling observed with the PE. The remainder of this paper is organized as follows: Section II describes aspects of solitary waves, specific wave details for the shallow-water environment which we model, and their impact on acoustic modes. Section III contains the derivation and evaluation of an expression which can determine mode-coupling likelihood for our specific ISW's. Sections IV-VII present and analyze results for the 
two-dimensional (2-D) propagation case, i.e., the case where the ISW wavevector and the acoustic horizontal wavevector are colinear. Specifically, Section IV shows PE propagation modeling results, including mode coupling. Section $\mathrm{V}$ shows sharp interface analytic mode-coupling results, which help diagnose the PE results. Section VI catalogs the propagation into various coupling regimes based on the physics. Section VIII shows results indicating that the 2-D model is satisfactory for a large range of horizontal angles of incidence of the acoustic waves on the ISW's. A summary concludes the paper.

\section{INTERNAL SOLITARY WAVES}

Solitary waves are hump-like single-crested waves which travel at a steady phase velocity and retain their shape as they propagate. Their properties and dynamics are described well by Lighthill [10], Witham [11], and Apel et al. [3]. Additional monographs provide more details and nuances.

These waves are solutions of a few related nonlinear equations [3], derivable through various means for either the deep-ocean or a shallow-water ocean waveguide. They share the characteristic of a balance between nonlinear steepening of the waves and the dispersive propagation of the various harmonic components of the waves, which tends to flatten them.

The Korteweg-de Vries ( KdV) equation describes the shape of solitons and their propagation for the shallow-water (longwave) situation. Two-layer systems are often used to derive analytic soliton shapes, sizes, and kinematics [2], [3], [12]. Features observed in the ocean do not always exactly match the analytic solutions, but features resembling them occur often enough to warrant comparison. Most of the observed features have the form of propagating, stable depressions of the thermocline (the interface in the two-layer limit).

The nonlinear equations have many solutions for interfacial ISW's. A few examples are given in a table by Bogucki and Garrett [12]. An example to compare with the shapes used for our simulations is the two-layer interfacial shallow-water ISW solution. Consider a system with upper layer of depth $h_{1}$, lower layer depth of $h_{2}$, associated densities $\rho_{1}$ and $\rho_{2}$, and $h_{2} \ll L$ so that the $\mathrm{KdV}$ equation is valid, where $L$ is the horizontal wave scale. The shallow-water ISW displacement of the boundary is

$$
\eta(x, t)=-a \operatorname{sech}^{2}\left[\left(x-c_{p} t\right) / L\right]
$$

with downward displacement when $h_{1}<h_{2}$. The phase speed is

$$
c_{p}=c_{L}\left(1+\frac{a}{2} \frac{h_{2}-h_{1}}{h_{1} h_{2}}\right)
$$

where the phase speed of an infinitesimally long and small wave is

$$
c_{L}=\left[g \frac{\rho_{2}-\rho_{1}}{\rho_{2}} \frac{h_{1} h_{2}}{h_{1}+h_{2}}\right]^{1 / 2} .
$$

The relationship between amplitude and horizontal wave scale is

$$
a L^{2}=\frac{4}{3} \frac{\left(h_{1} h_{2}\right)^{2}}{h_{2}-h_{1}} .
$$

As written, the wavevector of the solitary wave points in the $x$ direction, a convention throughout this paper.

For reasons stated in the introduction, we extend our analysis to additional $(a, L)$ parameter pairs not fitting the above relation. This allows us both to pin down acoustic coupling mechanisms by observing more parameter pairs and to model oblique sound incidence which would stretch waves scales along an acoustic path.

\section{A. Single Soliton Effects}

An initially pulse-like thermocline depression has been observed to separate into independently propagating solitons [6]. Each soliton has a characteristic amplitude, horizontal scale, and phase speed. Together they compose a soliton packet. Since each soliton in the packet propagates at a different phase speed, the spacing between solitons will change as the packet propagates. Therefore, the horizontal scales of the solitons and the spacing between them within a packet are both variable and can be dissimilar. For this reason, acoustic propagation analyses which assume a periodic internal wave structure within a soliton packet [5] may be idealistic. To provide a rigorous first step in mode-coupling analysis, we investigate the propagation of acoustic modes through a single soliton rather than through a packet of solitons.

\section{B. Model Environment for Acoustic Simulation}

We model acoustical propagation through waves of various $a$ and $L$ superimposed on a background three-layer stratified system. The calculations in this manuscript neglect the weak functional dependence of sound speed $c(T, S, P)$ on pressure $P$, and assume constant temperature $T$ and salinity $S$ in each of the top and bottom layers. The resultant constant soundspeed in the top and bottom layers simplifies the derivation of adiabatic invariance expressions in Section III.

Fig. 1 shows the shape of the wave and the various defining parameters. The water depth is $50 \mathrm{~m}$. The sound speed in the bottom is $c=1673.3 \mathrm{~m} / \mathrm{s}$ and the density of the bottom is 2000 $\mathrm{kg} / \mathrm{m}^{3}$, consistent with sand and/or sand with some gravel. The top water layer has thickness $h_{1}=10 \mathrm{~m}$ and sound speed $c_{1}=$ $1522 \mathrm{~m} / \mathrm{s}$, consistent with temperature $21.4{ }^{\circ} \mathrm{C}$ and salinity 32 psu. The lower layer has thickness $h_{2}=30 \mathrm{~m}$ and sound speed $c_{2}=1494 \mathrm{~m} / \mathrm{s}$, consistent with temperature $12.2{ }^{\circ} \mathrm{C}$ and salinity $32 \mathrm{psu}$. A constant-gradient layer lies between the isovelocity layers. The attenuation in the bottom is set to either 0.0 or $0.7 \mathrm{~dB}$ per wavelength, consistent with losses for 400 $\mathrm{Hz}$ in a sandy bottom. All the results presented here are for the lossless situation. The zero-loss study allows investigation of the evolution of mode coefficients due only to coupling. The results for the lossy bottom case were qualitatively similar to those for the zero loss case and are not shown. In both cases, a pressure release floor was positioned at a depth of $200 \mathrm{~m}$ below the sea surface.

\section{The Mode Representation}

Acoustic mode shapes are vertical basis functions such that in a range-invariant waveguide the acoustic pressure at range 


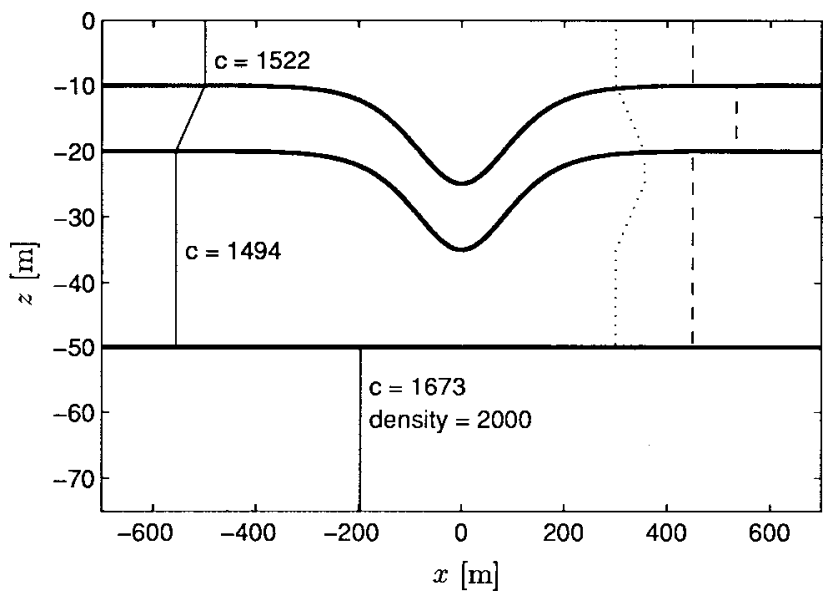

Fig. 1. The basic environment is shown. There are two isothermal mixed layers and a constant-gradient transition layer. The transition layer has constant thickness. The upper layer has (unperturbed) thickness $h_{1}=10 \mathrm{~m}$ and $c_{1}=$ $1522 \mathrm{~m} / \mathrm{s}$; the lower water layer has thickness $h_{2}=30 \mathrm{~m}$ and $c_{1}=1494$ $\mathrm{m} / \mathrm{s}$. The bottom terminates at depth $200 \mathrm{~m}$, has $c=1673.3 \mathrm{~m} / \mathrm{s}$, density 2000 $\mathrm{kg} / \mathrm{m}^{3}$, and attenuation either 0.0 or $0.7 \mathrm{~dB}$ per acoustic wavelength at 400 Hz. An example soliton with depression $\eta=a \operatorname{sech}^{2}(x / L)$ is shown, with $a=15$ and $L=125$. The dotted profile to the right is the actual sound-speed perturbation in the center of the ISW and the dashed line is the linearized estimate at the same point, $\eta$ times unperturbed $\partial c / \partial z$.

$x$ and depth $z$ has the separable form

$$
p(x, z)=\sum_{i} M_{i}(x) \phi_{i}(z) .
$$

The mode shapes $\phi_{i}(z)$ are functions of the vertical structure of the environment. Each mode coefficient $M_{i}(x)$ is determined by a scalar differential equation. In a range-varying waveguide, the mode shapes are functions of $x$ as well as $z$ and the set of mode coefficients is determined by a coupled vector differential equation [13].

Attempts have been made to use linear perturbation techniques [9] to model the change in mode shapes resulting from changes in the sound-speed profile. However, for vertical wave displacements more than a few meters, these techniques did not provide sufficiently accurate descriptions of the resulting changes in the mode shapes. Therefore, in this work, acoustic mode shapes are computed at all locations throughout the medium with the program FEMODE [14]. These can be computed for any stratification encountered and are calculated as frequently as required for accuracy. The shapes of a few acoustic modes are shown in Fig. 2. The rapid change of mode shapes within solitons will be shown to strongly influence mode coupling.

\section{Nonlinear Sound-Speed Perturbation}

A notable feature of soliton displacements of nearly isothermal or isovelocity layers is that the perturbed sound-speed field cannot be expressed in terms of a series expansion. Ordinarily, the perturbation for deep-ocean sound-speed fluctuations is expressed in the linearized form $\delta C=\eta(\partial C / \partial z)$. This is implicit in the acoustic mode-coupling analysis for internal waves by Dozier and Tappert [15]. This is clearly violated when the local vertical sound-speed gradient changes significantly over a depth interval of $\eta$. Examples include situations

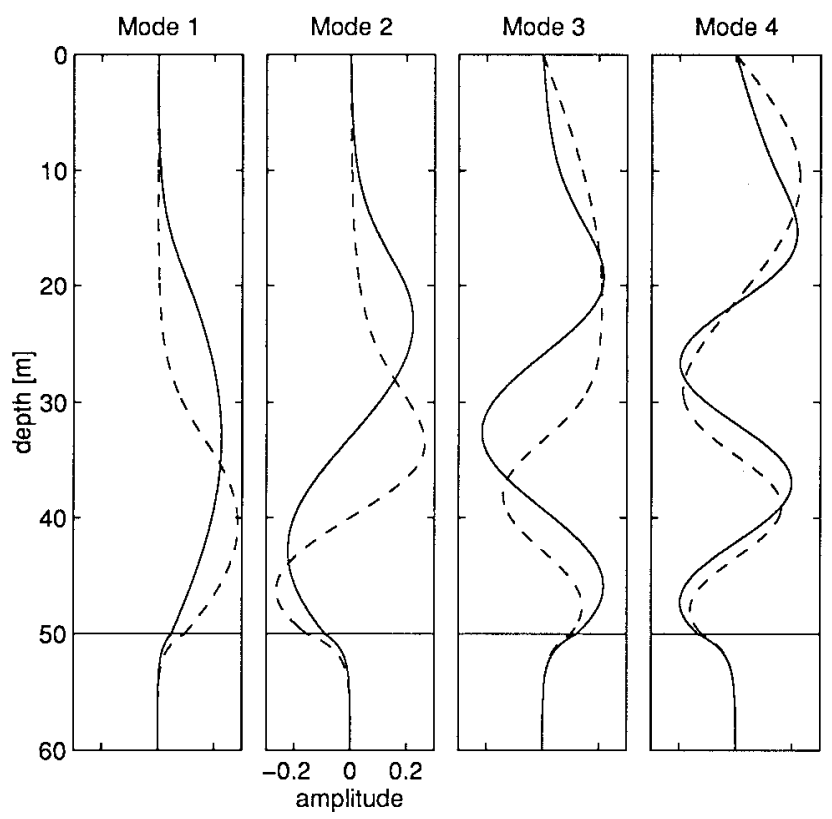

Fig. 2. Acoustic mode shapes for the three-layer system of Fig. 1 are shown. The solid lines are the modes away from the ISW, the dashed lines are the modes in the center of the ISW, with $\eta=15-\mathrm{m}$ displacement.

where the water of the upper layer intrudes to depths ordinarily associated with the deep layers, a common occurrence in the presence of ISW's. Fig. 1 illustrates this.

\section{CRITERION FOR ADIABATIC INVARIANCE}

Propagation through a medium having a range-dependent sound-speed profile can lead to energy transfer between modes, termed mode coupling. The total absence of mode coupling is termed adiabatic propagation, and the absence of direct mode coupling between two particular modes is termed adiabatic invariance. Milder [4] developed an expression to determine the environmental regimes in which mode coupling or adiabatic invariance will hold for selected mode pairs. Evaluation of the expression will show that the domain of each of the two regimes depends greatly on which mode pairs are considered.

The expression contains a depth integral with a triple product integrand. The factors are the two modal shapes under consideration and the horizontal derivative of a sound velocity term. Clearly, each of these factors are functions of range and profile gradients. The criterion for adiabatic invariance of modes $i$ and $j$ is [4]

$$
\left|\frac{X_{i j}(x(s))\left(\int \phi_{i}(x(s)) \frac{\partial U(x(s), z)}{\partial s} \phi_{j}(x(s)) d z\right)}{\Delta E_{i j}(x(s))}\right| \ll \pi .
$$

The variable $s$ denotes the path of propagation of the acoustic field. The direction of ISW propagation is given by $x$. The numerator on the left-hand side of the expression represents the effective change in $U(x, z) \equiv \omega^{2}\left(n_{o}^{2}-n(x, z)^{2}\right)=$ $k_{o}^{2}-k(x, z)^{2}$ (a wavenumber-squared-like quantity) integrated over depth, occurring over a classical ray period $X_{i j}(x)=$ $2 \pi / \Delta k_{i j}(x)$ where $\Delta k_{i j}(x) \equiv\left|k_{i}(x)-k_{j}(x)\right| . X_{i j}$ is also the mode interference length. $n \equiv 1 / c$ is the refractive index and $\omega$ is the acoustic frequency. The denominator 
represents the energy-level spacing of the modes in the terminology of Milder, a name derived from the analogous quantity in the quantum-mechanical Schrödinger equation analogy. The energy-level spacing is $\Delta E_{i j}(x) \simeq-2 k_{o} \Delta k_{i j}(x) \simeq$ $-4 \pi k_{o} / X_{i j}(x)$. Here, $k_{o} \equiv \omega / c_{o}$ where $c_{o}=\omega n_{o}$ is the nominal depth-averaged sound speed. Equation (6) states that the ratio of this depth-weighted effective change in $U(x, z)$, occurring over length scale $X$, to the energy-level spacing between two modes must be small in order for the propagation to be adiabatic.

Defining $\theta(x) \in[-\pi / 2, \pi / 2]$ as the angle between the soliton and acoustic wave vectors and suppressing the functional dependence of $x$ on $s$, (6) can be rewritten as

$$
\cos \theta(x)\left|\frac{X_{i j}(x)\left(\int \phi_{i}(x) \frac{\partial U(x, z)}{\partial x} \phi_{j}(x) d z\right)}{\Delta E_{i j}(x)}\right| \ll \pi
$$

which can be simplified to

$$
\cos \theta\left|\frac{\int \phi_{i} \frac{\partial U(x, z)}{\partial x} \phi_{j} d z}{k_{O} \Delta k_{i j}^{2}}\right| \ll 1
$$

The dependence of $\phi$ on $x$ and $z$ and the dependence of $\theta$ and $\Delta k_{i j}^{2}$ on $x$ has been suppressed.

In the three-layer ISW model described above, (8) can be operated on to give a reduced expression in terms of the derivative $|d \eta(x, t) / d x|$. We can write

$$
\frac{\partial U(x, z)}{\partial x}=2 \omega^{2} c^{-3}(x, z) \frac{\partial c(x, z)}{\partial x} .
$$

Let $z_{u}$ and $z_{l}$ be the nominal upper and lower boundaries, respectively, of the middle transition layer (Fig. 1), $\Delta_{Z}=$ $z_{l}-z_{u}$ be the width of the middle transition layer, and $\Delta_{C}=c_{2}-c_{1}$. Our three-layer environment can be written as

$$
c(x, z)=\left\{\begin{array}{l}
c_{1}, \text { for } z \geq z_{u}+\eta(x) \\
c_{1}+\left(z-\left(z_{u}+\eta(x)\right)\right) \frac{\Delta_{C}}{\Delta_{Z}} \\
\quad \text { for } z_{u}+\eta(x)>z \geq z_{l}+\eta(x) \\
c_{2}, \text { for } z<z_{l}+\eta(x)
\end{array} .\right.
$$

Differentiating with respect to $x$ and substituting into (9) yields

$$
\begin{aligned}
& \frac{\partial U(x, z)}{\partial x} \\
& = \begin{cases}0, & \text { for } z \geq z_{u}+\eta(x) \\
-2 \frac{d \eta(x)}{d x} \omega^{2} c^{-3}(x, z) \frac{\Delta_{C}}{\Delta_{Z}}, & \text { for } z_{u}+\eta(x)>z \geq z_{l}+\eta(x) . \\
0, & \text { for } z<z_{l}+\eta(x)\end{cases}
\end{aligned}
$$

Thus, the limits of integration in (8) can be set at $z_{u}+\eta(x)$ and $z_{l}+\eta(x)$ and the inequality can be expressed as

$$
\cos \theta\left|\frac{d \eta(x)}{d x}\right| \ll \frac{\Delta k_{i j}(x)^{2}}{U_{i j}(x)} \frac{\Delta_{Z}}{2 \omega c_{o} \Delta_{C}}
$$

where a weighted projection at the perturbed depths has been introduced

$$
U_{i j}(x)=\left|\int_{z_{l}+\eta(x)}^{z_{u}+\eta(x)} \phi_{i}(x, z) \phi_{j}(x, z) c^{-3}(x, z) d z\right| .
$$

Using the ISW shape given in (1) yields the expression

$$
\frac{d \eta(x)}{d x}=-\frac{2 a}{L} \operatorname{sech}^{2}(x / L) \tanh (x / L) .
$$

Finally, combining (11) and (13) yields the adiabatic criterion

$$
L \gg a \beta_{i j}(a, x / L) \cos \theta(x)
$$

where

$$
\beta_{i j}(a, x / L)=\frac{4 \omega c_{o} \Delta_{C}}{\Delta_{Z}} \frac{U_{i j}(a, x / L) \operatorname{sech}^{2}(x / L) \tanh (x / L)}{\Delta k_{i j}^{2}(x / L)} .
$$

The functional dependence of $\beta$ on $a$ and $L$ reflects the dependence of $\eta, \phi_{i}$, and $\Delta k_{i} j$ on $a$ and $L$. For given $i$ and $j, \beta$ depends on $x / L$ and has the same shape for all $L$.

For given $a$ and $L$ values, mode pairs $i$ and $j$ with larger values of $\beta_{i j}(a, x)$ are more likely to exhibit coupling. Physically, the expression states that mode $i, j$ coupling is more likely if $\Delta k_{i j}(x)$ is small or if the weighted projection of the mode $i$ onto mode $j$ over the transition layer is large. This second criterion is intuitive since the transition layer is the only place in the water column where the horizontal gradient of the sound-speed is not zero. As different $i j$ pairs are considered, the weighted projection varies much more than $\Delta k_{i j}(x)$, meaning that the coupling is more directly controlled by the mode shapes at the depths with appreciable horizontal gradient of sound speed.

The coupling expressions are evaluated at $\omega / 2 \pi=400$ and $200 \mathrm{~Hz}$ for our three-layer model. Fig. 3 shows 400$\mathrm{Hz} \beta_{i j}(a, x / L)$ as $x$ moves toward the center of an ISW depression at $x=0$. The maximum of $\beta$, where coupling is strongest, is generally at $x / L<0.7$. This is where the thermocline has maximum slope [(13)] [also shown in Fig. 3]. Fig. 3(a) and (b) show that adjacent modes interact more strongly than nonadjacent modes. Fig. 3(c) shows that the mode 3 to 4 interaction is quite strong relative to the mode 1 to 2 or mode 2 to 3 interaction, so that these modes require the largest $L$ value for adiabatic invariance. This conclusion is supported by numerical results (Section IV). The coupling of adjacent modes is more probable because the mode shapes overlap well in the thermocline region where $\partial U / \partial x$ is high.

Fig. 4 shows maxima (as functions of $x / L$ ) of the nonlinear functions $a \beta(a, x / L)$ for various mode interaction pairs at 400 $\mathrm{Hz}$. Recall that modes are predicted to interact if $L$ is not significantly greater than the plotted $a \beta$ values. Fig. 5 shows $200-\mathrm{Hz} a \beta$, which generally show transition from coupled to adiabatic propagation at shorter $L$ than for $400 \mathrm{~Hz}$.

Table I shows maxima of $400-\mathrm{Hz} a \beta$ for a sech-shaped ISW of $a=15 \mathrm{~m}$. The most stringent $L$ constraints are for mode pairs $3 / 4$ and $4 / 5$, which would couple for $L$ of many hundreds of meters. The next most stringent constraints are for $2 / 3$ and $3 / 5$.

Table II shows the mode interference lengths $2 \pi / \Delta k_{i j}$ for the modes of the background stratification. These are greater than $L$ for the energetic low-order modes and do not seem to be closely associated with coupling propensity (Table I). They remain larger than $200 \mathrm{~m}$ when computed with any set of mode shapes applicable within the ISW trough. These are less 

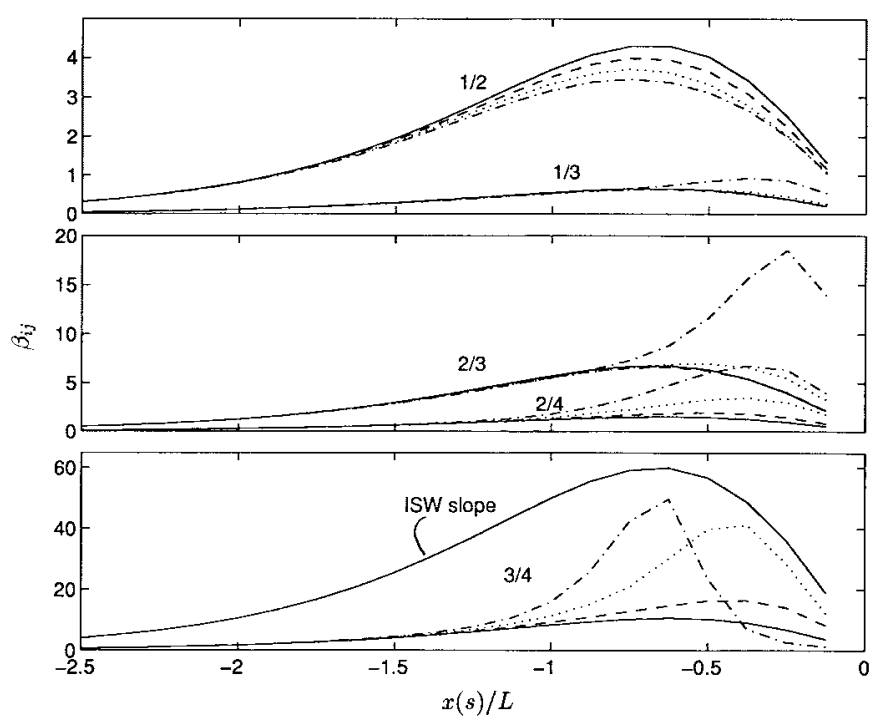

Fig. 3. (a) The coupling strength variability at $400 \mathrm{~Hz}$ in sech-type ISW's, $\beta_{i j}(a, x / L)$, is plotted for mode pairs $i=1, j=2,3$, and various amplitudes. Zero is the trough of the ISW. The key is $a=5$ (solid line), 10 (dashed line), 15 (dotted lines) and $20 \mathrm{~m}$ (dot-dash). The adjacent mode $1 / 2$ coupling is always stronger than $1 / 3$. (b) The same function $\beta$ is plotted for $i=2$ and $j=3,4$, with the line types indicating the $a$ value as in (a) Again, the adjacent mode coupling (2/3) is stronger than the nonadjacent. (c) The strength function $\beta_{34}(a, x / L)$ is plotted for the same $a$ values, indicated by line type as in (a). The 15- and 20-m amplitude solitary waves exhibit a strong $3 / 4$ mode coupling tendency $\left(\beta_{34}>40\right)$ relative to other pairs that were examined. The functions tend to peak at $x / L<0.7$. The functions often have the same shape as the thermocline slope $|d \eta / d x|$, the shape of which is shown in (c).
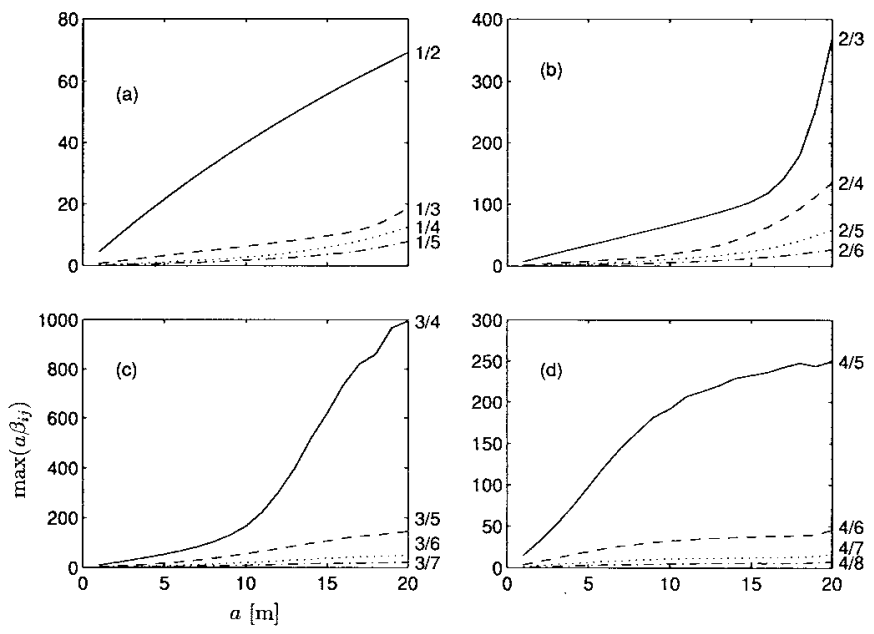

Fig. 4. The peak values, with respect to $x$, of the nonlinear functions $a \beta_{i j}(a, x / L)$ for $400 \mathrm{~Hz}$ are shown as functions of $a$. Each frame has a particular value of $i$ ranging from 1 to 4 and variable $j$ ranging from $i+1$ to $i+3$. Solitons with scale lengths $L \gg a \beta_{i j}$ meet the adiabatic criterion, those near $a \beta_{i j}$ or below are predicted to couple. Mode pairs $3 / 4$ and $4 / 5$ show coupling tendencies extending to the greatest scales, while other pairs such as $1 / 3$ and $1 / 4$ would couple only at very small $L$.

variable than $a \beta$ (Table I), showing the sensitivity of coupling to $U_{i j}$ variations rather than $\Delta k_{i j}$.

\section{PE COMPUtational Propagation}

The FEPE program generates a solution for the acoustical field radiating outward from a source using a wide-angle PE
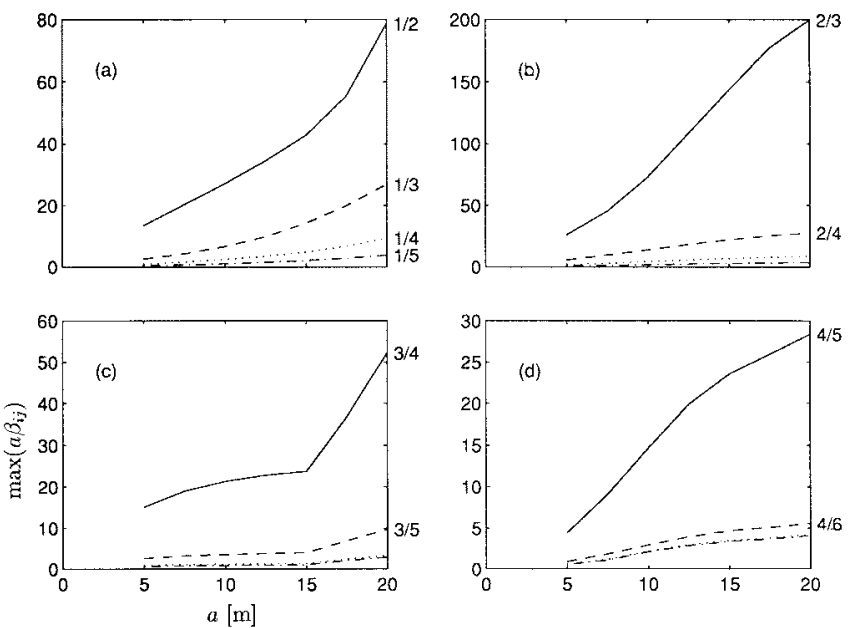

Fig. 5. Similar to Fig. 4, except for $200 \mathrm{~Hz}$. The line types are as labeled in Fig. 4: (a) The mode $1 / j$ adiabatic boundaries $\left(a \beta_{i j}\right)$ are similar to those of $400 \mathrm{~Hz}$. (b) The $2 / j$ boundaries are lower than for $400 \mathrm{~Hz}$. (c) The mode $3 / j$ boundaries are very low, much different than the $400-\mathrm{Hz}$ case, especially $3 / 4$

(d) The $4 / j$ boundaries are very low, so adiabatic transmission is expected for virtually all $L$.

TABLE I

MAXimum VALUES OF FUnCTION $a \beta_{i j}$ Within $a=15$ ISW's

\begin{tabular}{ccccccccc}
\hline- & 1 & 2 & 3 & 4 & 5 & 6 & 7 & 8 \\
1 & - & 56 & 10 & 6.0 & 4.0 & 2.2 & 1.4 & 0.9 \\
2 & - & - & 104 & 52 & 24 & 13 & 6.9 & 4.1 \\
3 & - & - & - & 619 & 106 & 37 & 16 & 8.3 \\
4 & - & - & - & - & 233 & 37 & 12 & 5.6 \\
5 & - & - & - & - & - & 121 & 24 & 8.8 \\
6 & - & - & - & - & - & - & 68 & 14 \\
7 & - & - & - & - & - & - & - & 59 \\
8 & - & - & - & - & - & - & - & -
\end{tabular}

The results are not dependent on $L$.

TABLE II

MOde INTERFERENCE LENGTHS FOR THE MOdES of the Background Medium, $2 \pi /\left(k_{i}-k_{j}\right)$

\begin{tabular}{ccccccccc}
\hline- & 1 & 2 & 3 & 4 & 5 & 6 & 7 & 8 \\
1 & - & 1104 & 425 & 242 & 172 & 132 & 102 & 80 \\
2 & - & - & 692 & 310 & 203 & 150 & 113 & 86 \\
3 & - & - & - & 561 & 288 & 191 & 134 & 99 \\
4 & - & - & - & - & 591 & 290 & 176 & 120 \\
5 & - & - & - & - & - & 570 & 252 & 150 \\
6 & - & - & - & - & - & - & 451 & 203 \\
7 & - & - & - & - & - & - & - & 369 \\
8 & - & - & - & - & - & - & - & -
\end{tabular}

approximation [14], [16]. In interpreting the results which follow, it is important to note two characteristics of the PE approximation. The first is that the PE approximation models only forward propagation, so any possible backscattering is not accounted for. The same holds true for the SIA described in Section V. The second characteristic is that the PE implementation is $2-\mathrm{D}$, so the environment is assumed to be radially 
symmetric about the source location, and the acoustic field is calculated radially outward from the source. Thus, there is no horizontal refraction of the field. The applicability of these results to three-dimensional (3-D) environments is discussed in Section VIII.

The acoustic field was calculated at a depth increment of 0.1 $\mathrm{m}$ up to a depth of $200 \mathrm{~m}$ (i.e., $150 \mathrm{~m}$ into the bottom) and a range increment of $0.75 \mathrm{~m}$. The expansion parameter in the code, NPADE, was set to 2 . Mode filtering using the computed mode shapes at 1-m-depth increments up to a depth of $100 \mathrm{~m}$ was used to estimate the modal content of the acoustic field at each range $(x)$ of interest. This 1 -m sampled mode filter results in a maximum crosstalk between modes of $-67 \mathrm{~dB}$ for the modes considered. The model coefficients at each range are normalized so that $\sum_{i}\left|c_{i}(x)\right|^{2}=1$ where $c_{i}$ is the complex amplitude of the $i$ th mode.

The particular conditions for the simulations are that the acoustic field begins propagating at range of $x=-2100$ $\mathrm{m}$ and propagates to range of $x=2100 \mathrm{~m}$. An internal solitary wave with amplitude $a$ and horizontal scale $L$ is assumed to be centered at range of $x=0 \mathrm{~m}$ (Fig. 1). A quasistatic approximation is used, meaning that the ISW amplitude, horizontal scale, and location are assumed fixed over the time required for the acoustic signal to propagate through the ISW. The following subsections examine the modal content of the acoustic field as a function of range $(x)$, ISW horizontal scale $(L)$, and ISW amplitude $(a)$.

\section{A. Modal Content versus Range}

The first set of results show the evolution of the modal content of the acoustic field at $400 \mathrm{~Hz}$ as it propagates through solitons having $a=15 \mathrm{~m}$ and $L=25,75,250$, and $700 \mathrm{~m}$. Fig. 6 shows the test scenario and results for the case where $L=75 \mathrm{~m}$ and the initial acoustic field is composed entirely of mode 1. Fig. 7 shows results for all $L$ values for the initially mode 1 field. Fig. 8 shows results for the initially mode 3 field.

Several points are worth noting. For both the mode 1 and mode 3 cases, the amount of energy remaining in the original mode when the acoustic signal reaches the midpoint of the soliton $(x=0 \mathrm{~m})$ increases with $L$. Thus, the shorter steeper solitons induce more mode coupling in their first half than do the longer flatter solitons. This is expected since the horizontal gradient of the sound-speed profile is smaller for the longer solitons. Notice also that reasonable coupling exists at much longer soliton scales for the coupling from mode 3 to mode 4 than for coupling from mode 1 to other modes. This is in agreement with the adiabatic criterion calculations (Fig. 4 and Table I).

A monotonic relationship between the amount of coupling and the soliton scale is not seen for the net coupling through the total soliton. In particular, much of the coupling which takes place in the first half of the soliton for $L=25 \mathrm{~m}$ is undone or cancelled in the second half of the second half of the soliton. This phenomena is referred to as cancellation.

Coupling and uncoupling also occurs for $L=250 \mathrm{~m}$ when the initial acoustic field is composed entirely of mode 3 . The uncoupling, when it appears in solitons of scales that are longer

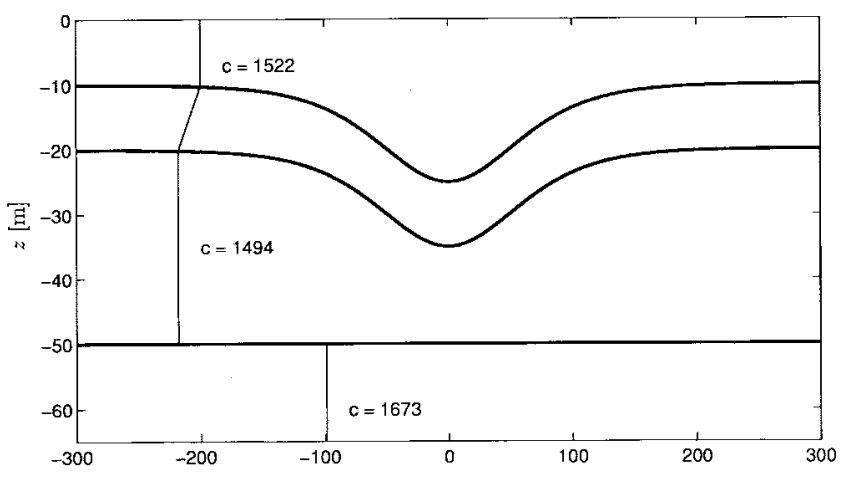

(a)

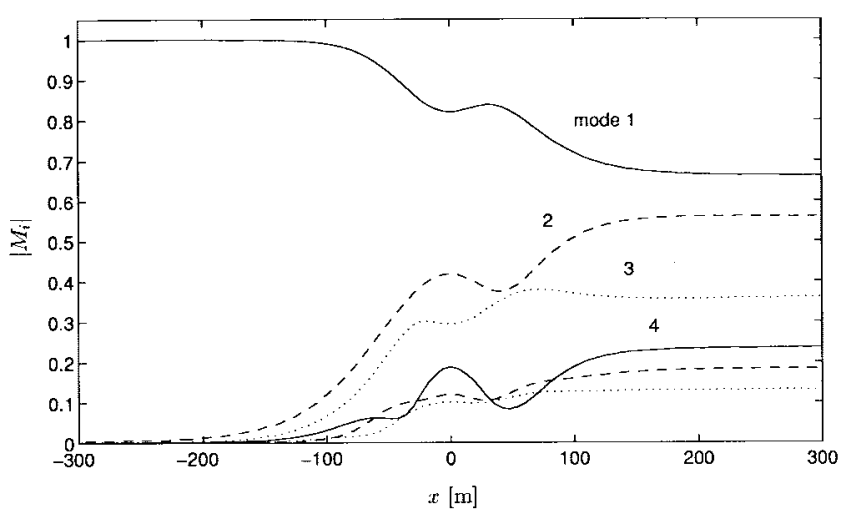

(b)

Fig. 6. Numerical PE solution of the wave equation shows interesting mode coupling behavior. Propagation through (a) $L=75 \mathrm{~m}, a=15-\mathrm{m}$ soliton yields (b) the modal decomposition. The energy is contained in mode 1 until the soliton is encountered. It couples into a variety of modes until the trough is reached, then energy briefly couples back into the original configuration. Coupling out of mode 1 resumes as the tail of the soliton is traversed, with a net loss of over $50 \%$ of the energy to other modes.

than the cancellation regime, is referred to as transparent resonance.

\section{B. Modal Content versus Soliton Horizontal Scale $(L)$}

The second set of $400-\mathrm{Hz}$ results show the modal content of the acoustic field as a function of $L$, with $a=15$ as before. Fig. 9 shows the modal content at the midpoint of the soliton $(x=0)$ for the cases where the initial acoustic field is composed entirely of modes 1,2 , and 3, respectively. Fig. 10 shows the analogous results at the endpoint of the propagation region $(x=2100 \mathrm{~m})$.

The midpoint results show a monotonic increase in the energy remaining in the initial mode (i.e., a decrease in the amount of mode coupling) as $L$ is increased. As predicted in Section III and shown in the previous section, the coupling at long $L$ is strongest for the initial field composed entirely of mode 3 . The endpoint results clearly show the cancellation and transparent resonance phenomena. In all cases, the endpoint results show minimal net coupling for very small $L$ ( $L \leq 20$ $\mathrm{m})$, but the midpoint results show significant coupling at the same $L$. For initial mode 3, the results show a significant local minimum in coupling strength at $L$ of roughly $225 \mathrm{~m}$ which are significantly longer than those of the cancellation regime. However, significant midpoint coupling occurs at $225 \mathrm{~m}$. This local minimum is a transparent resonance. 

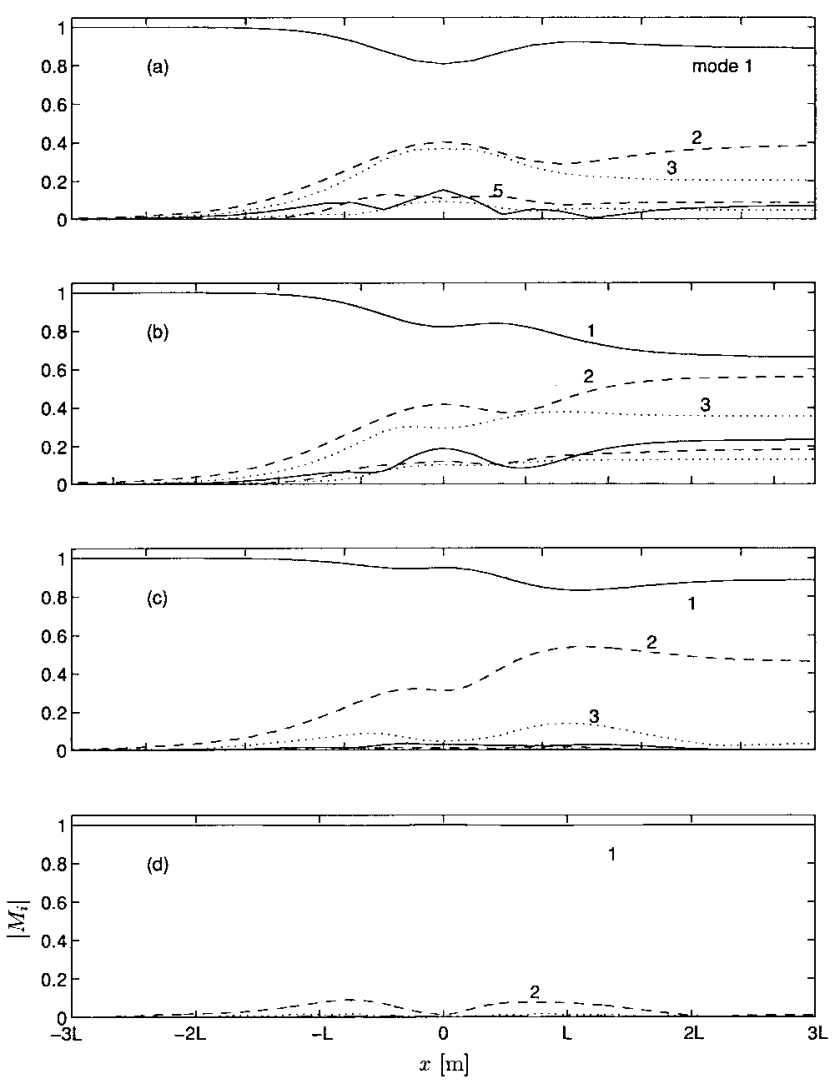

Fig. 7. PE computations of mode amplitude versus position within ISW's are shown for ISW's having $a=15 \mathrm{~m}$ and $L=25,75,250$, and $700 \mathrm{~m}$ [(a)-(d)]. The acoustic frequency is $400 \mathrm{~Hz}$, with a lossless bottom. Initial energy is in mode 1 . Modes 1 and 4 are shown with solid lines, modes 2 and 5 with dashed, and modes 3 and 6 with dotted. Coupling is the strongest at $L=75 \mathrm{~m}$, with most energy going into modes 2 and 3 .

\section{Modal Content versus Soliton Amplitude}

The modal content of the acoustic field at the midpoint of the soliton and the endpoint of the propagation region was computed as a function of the amplitude $(a)$ of the soliton. The horizontal scale of the soliton was fixed at $L=100$ m. Fig. 11 shows the midpoint results for the case where the initial acoustic field is composed entirely of modes 1-4. Fig. 12 shows the analogous endpoint results.

The midpoint results show two things. There is a monotonic increase in the amount of mode coupling as $a$ is increased, as expected. Also, there is a change in the distribution of coupled energy in the modes other than the incident mode as a function of $a$. The endpoint results for incident modes 2 and 3 clearly show the onset of the transparent resonance phenomena. This manifests itself as a decrease in the amount of modal coupling as $a$ is increased beyond about 15 and $13 \mathrm{~m}$, respectively.

\section{THE SHARP INTERFACE APPROXIMATION}

The sharp-interface model of propagation through an ISW is an application of conventional one-way coupled-mode computation [17]. In this model, the smooth shape of the ISW thermocline depression is replaced with a tophat-like pair of steps, so that the domain is divided into three regions, with the outer two identical (Fig. 13). The approximated ISW is centered at $x=0$, and acoustic propagation is from negative
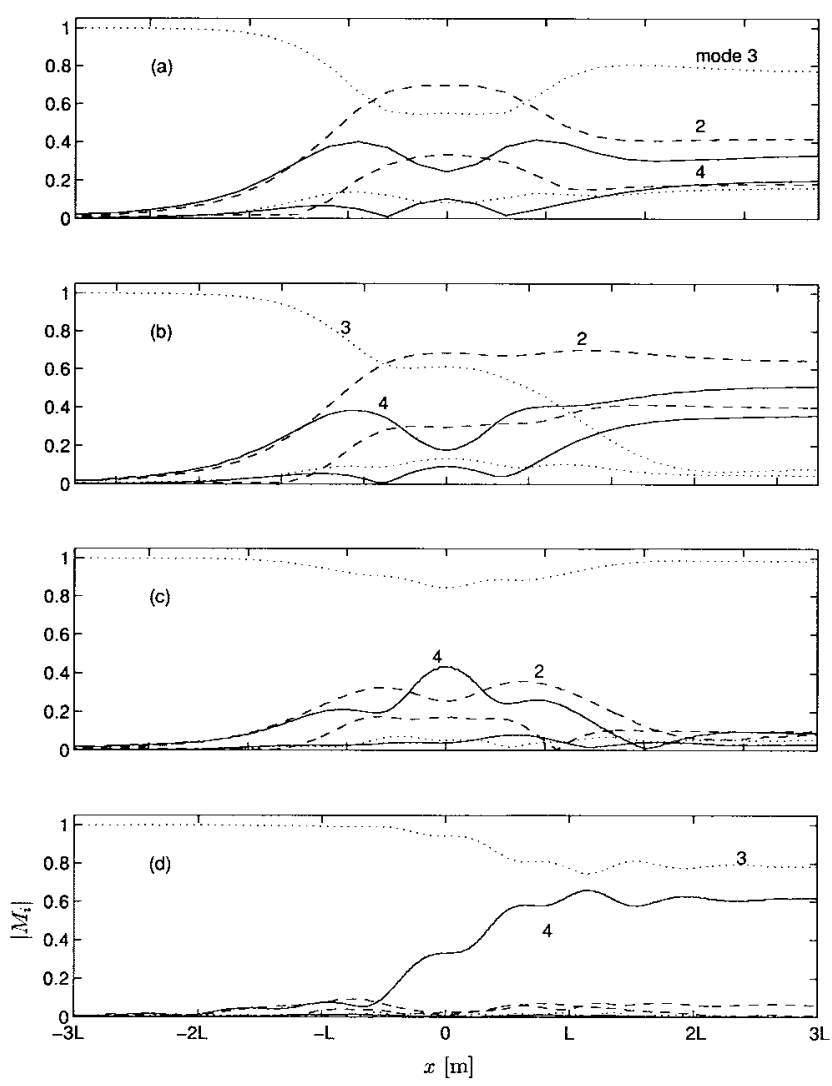

Fig. 8. PE computational results similar to those of Fig. 7 are shown, except that initial energy is in mode 3 . The lines are coded as before. The case for $L=700 \mathrm{~m}$ shows much transfer from mode 3 to mode 4 , unlike the initial mode 1 energy which behaved adiabatically (Fig. 7).

to positive $x$. Let $\mathrm{C}$ be the coupling matrix such that

$$
\mathbf{C}_{i j}=\int \rho^{-1}(z) \phi_{i}^{(1)}(z) \phi_{j}^{(0)}(z) d z .
$$

The vertical normal mode functions $\phi_{i}^{(0)}$ and $\phi_{i}^{(1)}$ are defined for the outer regions and the wave trough, respectively. Note that $\mathbf{C}$ is unitary, i.e., $\mathbf{C}^{t}=\mathbf{C}^{-1}$, so there is no backscattering of energy.

In the SIA, mode coupling as acoustic energy moves into the wave is written as

$$
\mathbf{M}_{1}\left(-L_{S}^{+}\right)=\mathbf{C M}_{0}\left(-L_{S}^{-}\right)
$$

where the vectors $\mathbf{M}_{0}$ and $\mathbf{M}_{1}$ denote the complex mode amplitude coefficients for the modes of the outer regions and wave trough, respectively. The indicated positions, $-L_{S}^{-}$and $-L_{S}^{+}$are just to the left and right of (acoustically before and after) the sharp interface at range $-L_{S}$. Removing the effect of wavefront spreading, the propagation within the central region (the wave trough) is given by a diagonal adiabatic propagation matrix

$$
\Lambda_{l l}=\exp \left[i k r_{l}^{(1)} 2 L_{S}\right]
$$

such that

$$
\mathbf{M}_{1}\left(L_{S}^{-}\right)=\Lambda \mathbf{M}_{1}\left(-L_{S}^{+}\right) .
$$

Here, $k r_{l}^{(1)}$ is the horizontal wave number of the $l$ th mode in the wave trough. (Make special note of the unorthodox twoletter symbol $k r_{l}$.) Coupling as energy exits the wave at the 

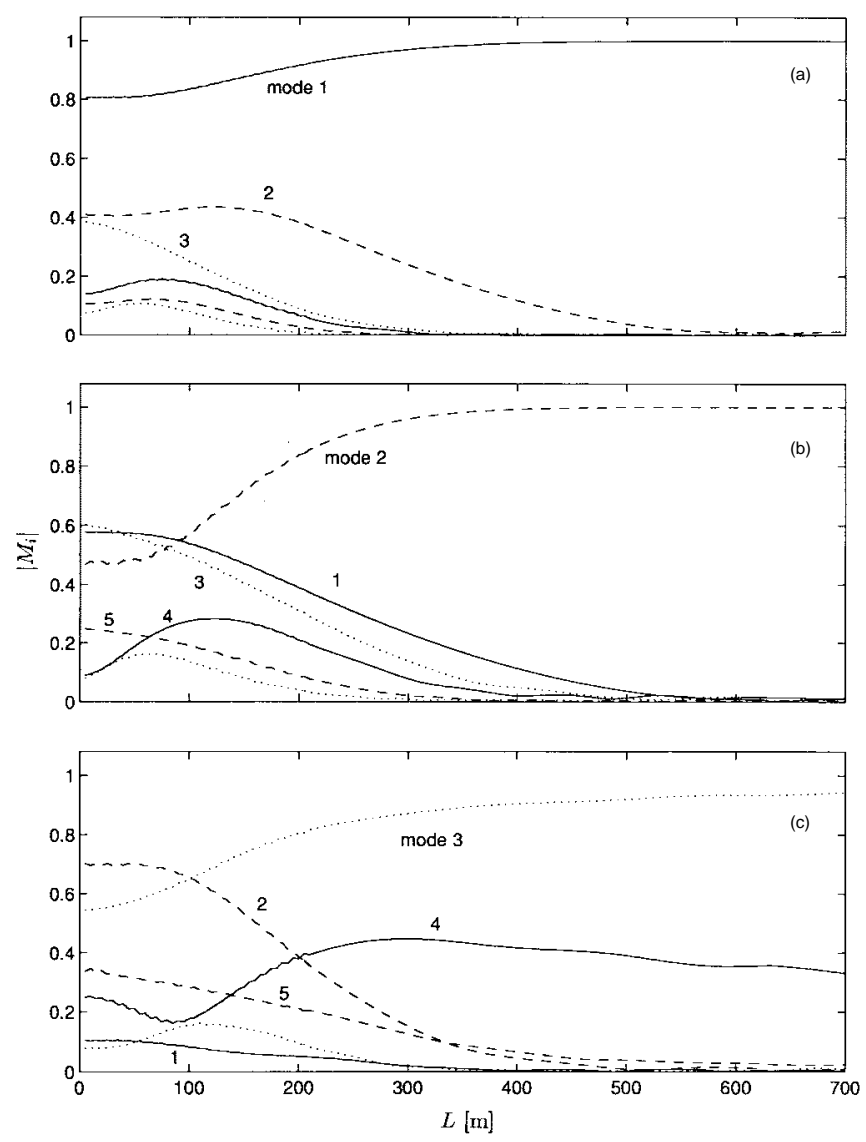

Fig. 9. PE computations of modal amplitude at the midpoint of ISW's of $a=15$ are shown as functions of ISW scale $L$. (a) The case with initial mode 1 energy is shown. (b) The case with initial mode 2 is shown. (c) The case with initial mode 3 is shown. In all cases, the maximum loss of energy from the initial mode occurs at the shortest $L$, corresponding to the steepest ISW. The coding of line type versus mode is similar to Figs. 6-8: modes 1 and 4 are solid, modes 2 and 5 are dashed, modes 3 and 6 are dotted.

second interface is given by

$$
\mathbf{M}_{0}\left(L_{S}^{+}\right)=\mathbf{C}^{t} \mathbf{M}_{1}\left(L_{S}^{-}\right) .
$$

A complete wave traverse is described by the linear operation

$$
\mathbf{M}_{0}\left(L_{S}^{+}\right)=\mathbf{C}^{t} \Lambda \mathbf{C M}_{0}\left(-L_{S}^{-}\right) .
$$

Note that if $L_{S}$ approaches 0 , then $\Lambda$ approaches the identity matrix. This yields the expected limiting case

$$
\begin{aligned}
\lim _{L_{S} \rightarrow 0} \mathbf{M}\left(L_{S}^{+}\right) & =\mathbf{C}^{t} \mathbf{C M}_{0}\left(-L_{S}^{-}\right) \\
& =\mathbf{C}^{-1} \mathbf{C M}_{0}\left(-L_{S}^{-}\right) \\
& =\mathbf{M}_{0}\left(-L_{S}^{-}\right) .
\end{aligned}
$$

The SIA representation of the propagation decomposes the propagation effects into three parts. The first is the coupling induced by the interface (represented by $\mathrm{C}$ ) which depends on only the amplitude $(a)$ of the soliton. The second is the propagation of the modes through the wave trough of the soliton which depends primarily on the horizontal scale $\left(L_{S}\right)$ of the soliton and only secondarily on $a$ though the functional dependence of $k r_{l}^{(1)}$ on $a$. The final part is the coupling induced at the exit interface.

Fig. 14 shows comparisons of the endpoint modal content versus horizontal scale for the PE simulations with those for the SIA, for initial acoustic fields composed of modes 1,2 , and
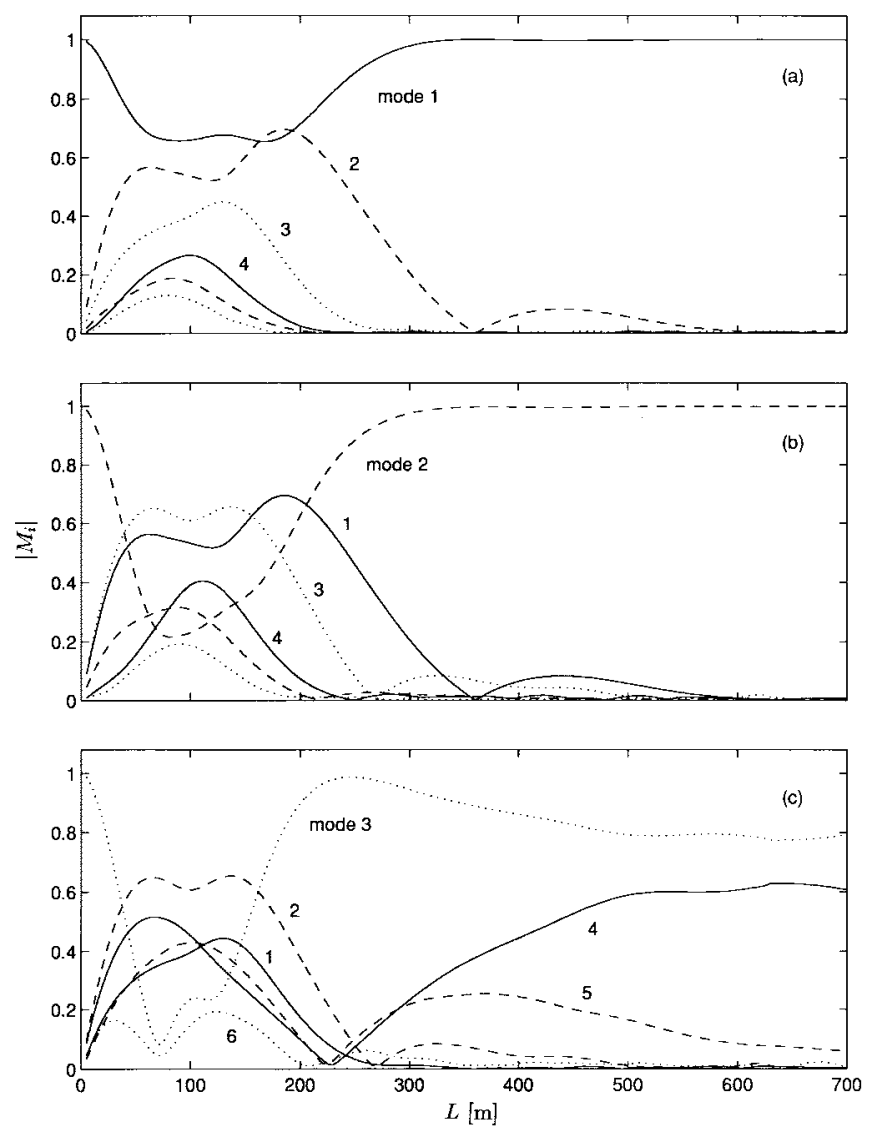

Fig. 10. PE computational mode amplitudes after complete traversal of ISW's are shown for $a=15 \mathrm{~m}$ ISW's of variable $L$. The cases are identical to those of Fig. 9. The shortest $L$ now shows little coupling because the coupling at midpoint, shown in Fig. 9, has been reversed. Maximum mode coupling now occurs at $50<L<175 \mathrm{~m}$.
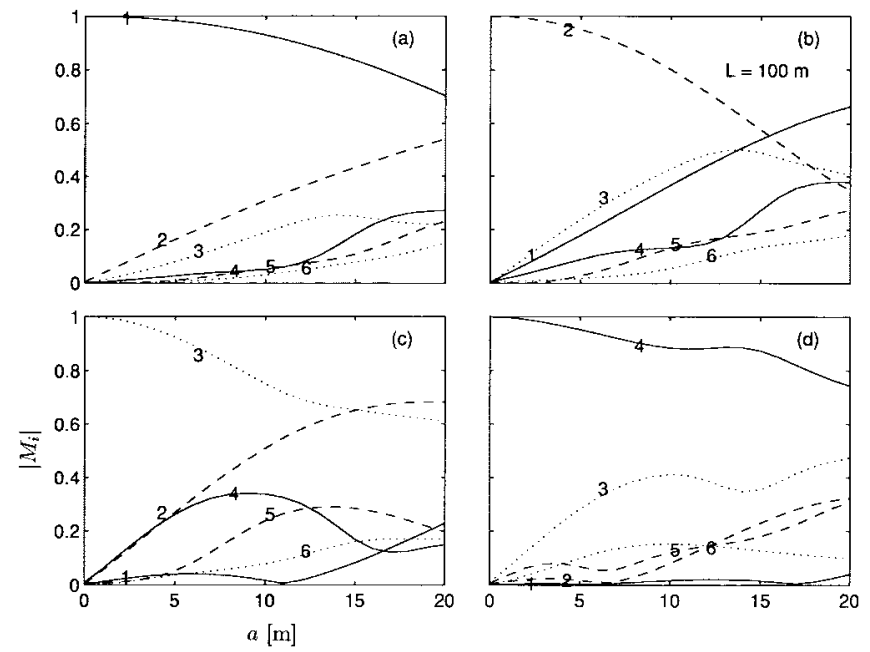

Fig. 11. PE computed mode amplitudes at the midpoint of ISW traversal are shown for variable $a$ ISW's having fixed $L=100$. (a) The initial mode 1 case is shown. (b) The initial mode 2 case is shown. (c) The initial mode 3 case is shown. (d) The initial mode 4 case is shown. Mode line type coding is the same as in previous figures. Coupling out of the initial mode increases with $a$.

3 , respectively. As with the PE results, the displayed quantities are the normalized magnitudes of the modal coefficients. In these cases, the amplitude of the sech-shaped wave used in the PE simulation is $15 \mathrm{~m}$ and the horizontal scale $(L)$ is as shown on the horizontal axis. The amplitude of the SIA 

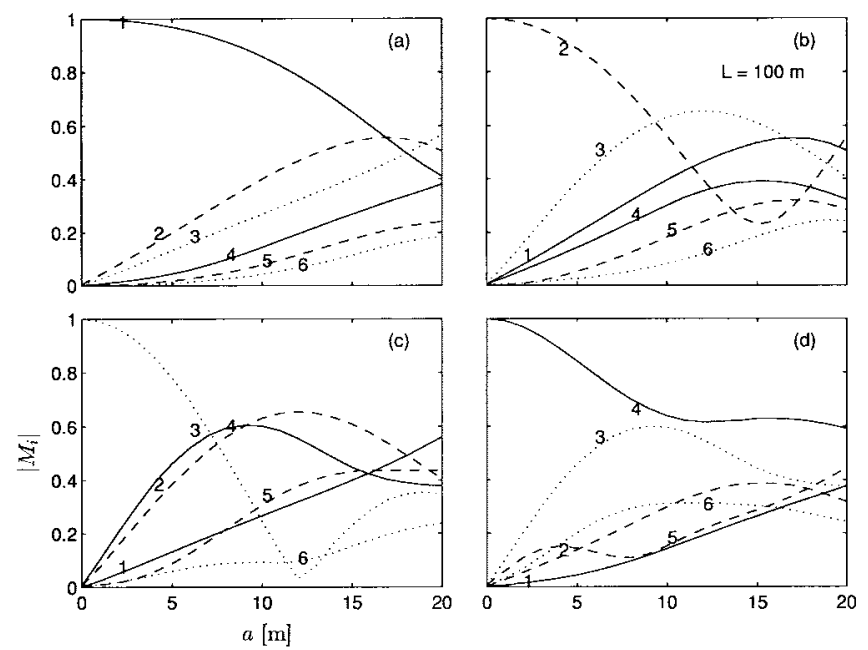

Fig. 12. PE computed mode amplitudes after complete ISW traversal are shown. The cases are analogous to those of Fig. 11, with $L=100 \mathrm{~m}$ and $a$ variable. The decreased coupling at higher $a$ for initial modes 2 and 3 , shown in (b) and (c), respectively, occurs because of reinforcing coupling at intermediate scales ( $a$ of 12-15 m) on both sides of the ISW trough which does not appear at high $a$.

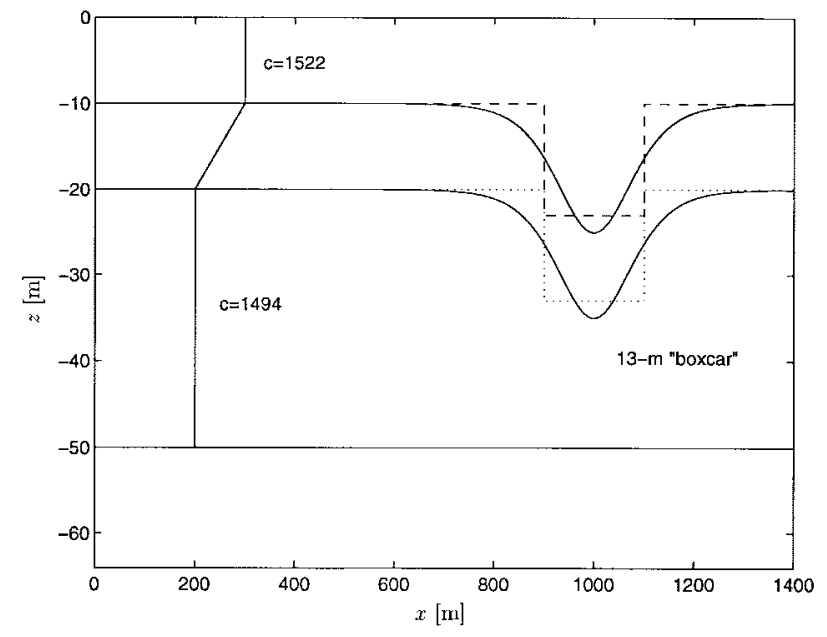

Fig. 13. The geometry of the SIA model is shown. An ISW of $a=15 \mathrm{~m}$, $L=100 \mathrm{~m}$ is shown. An SIA analog is a $13-\mathrm{m}$ inverted tophat of width $2 L$, with immediate coupling at each end of the trough. The three-layer model (Fig. 1) is otherwise unchanged.

wave is $12 \mathrm{~m}$ and the horizontal scale $\left(L_{S}\right)$ is as shown on the horizontal axis. The figures show reasonable qualitative agreement between the two sets of results for horizontal scales below about $200 \mathrm{~m}$. Results from the SIA approximation are robust over a range of sharp SIA wave depth and width scalings with respect to $a$ and $L$ of sech-type ISW's. The SIA was run with a variety of SIA scalings in addition to $L_{S}=L$ and slightly reduced SIA depth, giving qualitatively similar results varying slightly in detail. The comparison between full-ISW and SIA results for initial mode 3 is shown for an alternate case in Fig. 15. In this case, the amplitude of the SIA wave is $15 \mathrm{~m}$ and the horizontal scale $\left(L_{S}\right)$ equals 1.1 times that shown on the horizontal axis. This plot shows closer qualitative agreement than that in Fig. 14(c). The SIA results for this case qualitatively predict the presence of the transparent resonance.
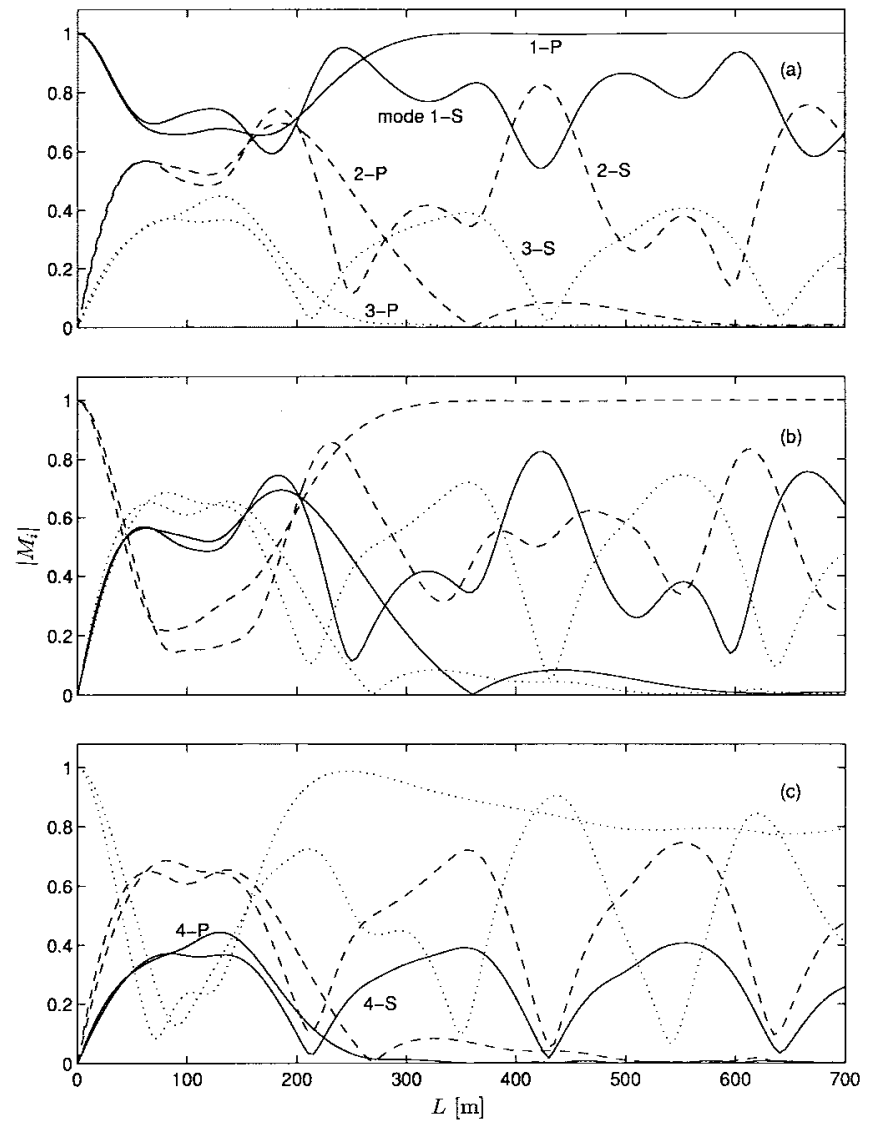

Fig. 14. SIA computed mode amplitudes after propagation through tophat ISW's of variable $L$ and fixed $a=12 \mathrm{~m}$ are shown, along with PE computational results of Fig. 10, for sech-type ISW, $a=15$. (a) The case of initial (before ISW) mode 1 energy is shown. Annotation 1-P indicates mode 1 from PE, 2-S indicates mode 2 from SIA, etc. Line types indicate mode number as in previous figures. (b) The case of initial mode 2 is shown, with line types the same as (a). (c) The case of initial mode 3 is shown. Unlike (a) and (b), mode 1 amplitude is omitted and mode 4 is shown because very little energy couples to mode 1 .

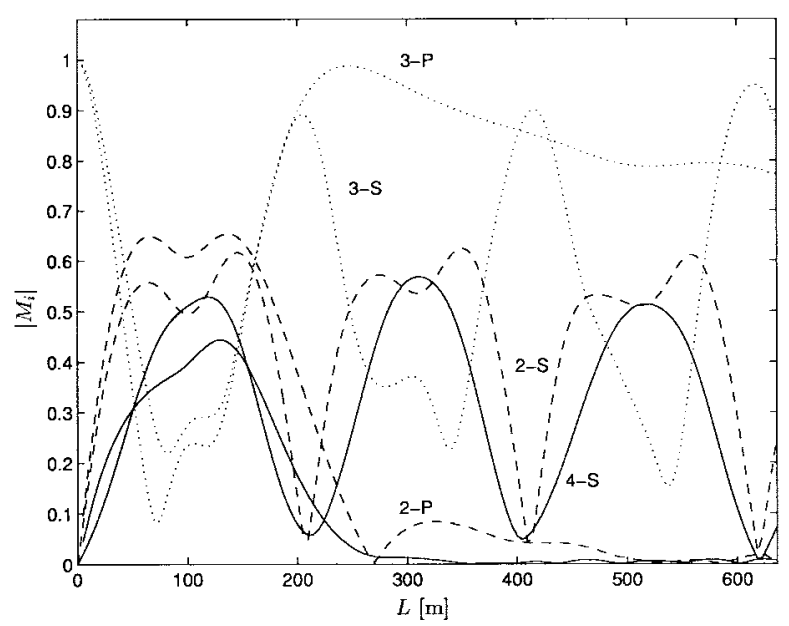

Fig. 15. Results similar to Fig. 14(c) are shown. The difference is that the SIA tophat-style ISW has depth $15 \mathrm{~m}$ rather than $12 \mathrm{~m}$, and the horizontal scale $L_{S}$ has increased from $L$ to $1.1 \times L$.

For horizontal scales greater than $200 \mathrm{~m}$, the SIA results show a continuing periodic pattern of strong coupling and weak coupling (transparent resonance) while the PE results 
do not. This divergence results from the inability of the SIA to model adiabatic propagation. The SIA is unsuitable for investigating the effects of lengthening horizontal scale and weakening horizontal gradients on coupling, but it is useful for modeling propagation at the smaller $L$ values with appreciable coupling.

\section{COUPLing Physics}

The physics which govern coupling in the cancellation, the first coupling, and the transparent resonance regimes can be understood by examining the results generated with the PE propagation program and the SIA formulas.

The SIA representation of the relationship between the modal coefficients before the acoustic field encounters the ISW and the modal coefficients after the acoustic field passes through the internal solitary wave is given by (21). Given that $\mathbf{C}$ is a unitary matrix, the matrix multiplication by $\mathbf{C}$ simply performs a transformation from the basis set of mode shapes $\phi_{i}^{(0)}$ to the basis set of $\phi_{i}^{(1)}$. This transformation is the essence of the sudden approximation to modal coupling. The matrix multiplication by $\Lambda$ independently propagates each of the modal coefficients. Finally, the matrix multiplication by $\mathbf{C}^{t}$ performs the inverse coordinate transformation of the first multiplication.

The SIA relationship between the pre-ISW coefficient for mode $i$ and the post-ISW coefficient for mode $j$ is given by

$$
\mathbf{M}_{0}\left(L_{S}^{+}\right)_{j}=\mathbf{C}_{j}^{t} \Lambda \mathbf{C}_{i} \mathbf{M}_{0}\left(-L_{S}^{-}\right)_{i}
$$

where $\mathbf{C}_{i}$ is the $i$ th column of the matrix $\mathbf{C}$ and $\mathbf{M}_{0}\left(-L_{S}^{-}\right)_{i}$ is the $i$ th element of the vector $\mathbf{M}_{0}\left(-L_{S}^{-}\right)$. Assume, for example, that upon entering the trough of the ISW, mode $i$ of the acoustic signal couples strongly into only modes $i, j, l, m$, and so on. That is, the only significant elements of $\mathbf{C}_{i}$ are the $i$ th, $j$ th, $l$ th, $m$ th, etc., elements. These modes will be referred to as the dominant internal modes. The number of dominant internal modes is four or less for all the situations we have studied. Then the exiting mode $j$ coefficient is

$$
\begin{aligned}
\mathbf{M}\left(L_{S}^{+}\right)_{j}= & \left(\mathbf{C}_{i j} \Lambda_{i i} \mathbf{C}_{i i}+\mathbf{C}_{j j} \Lambda_{j j} \mathbf{C}_{j i}+\mathbf{C}_{l j} \Lambda_{l l} \mathbf{C}_{l i}\right. \\
& \left.+\mathbf{C}_{m j} \Lambda_{m m} \mathbf{C}_{m i}+\cdots\right) \mathbf{M}\left(-L_{S}^{-}\right)_{i} .
\end{aligned}
$$

Equations (23) and (24) make it clear that the coupling from mode $i$ to mode $j$ by the passage of the acoustic signal through the ISW is the result of the coupling from mode $i$ to the dominant internal modes, the propagation of the dominant internal modes within the trough of the wave, and the final coupling from the dominant internal modes to mode $j$. Thus, the coupling from mode $i$ to $j$ depends not only on the relationship between these two modes (their mode shapes and horizontal wavenumbers) but also the relationships between each of these two modes and the dominant internal modes.

Figs. 9 and 10 illustrate the effects of the multiple coupling relationship. Fig. 9(c) indicates that for an ISW with $L=150$ $\mathrm{m}$, little energy is coupled from mode 3 to mode 1 while the acoustic signal passes though the first half of the ISW and significant energy is coupled from mode 3 to mode 2 in the same region. However, Fig. 10(c) indicates that modest energy is coupled from mode 3 to mode 1 while the acoustic signal passes through the entire ISW for the same value of $L$. An explanation can be found by looking at Fig. 9(a) which, at $L=150 \mathrm{~m}$, shows reasonable coupling from mode 1 to mode 2 in the first half of the ISW. This indicates strong interaction between the mode shapes $\phi_{1}^{(0)}$ and $\phi_{2}^{(1)}$. Thus, some of the energy in mode 1 which is present at $L=150$ in Fig. 10(c) can be accounted for by first coupling energy from mode 3 to mode 2 in the first half of the ISW and then from mode 2 to mode 1 in the second half of the wave. Evaluating the coefficients of the $\mathbf{C}$ matrix used in the SIA for this amplitude wave supports this conclusion.

Since the SIA expression describing the net mode coupling through the ISW is given by (24), the behavior of $\mathbf{C}$ and $\Lambda$ as a function of $a$ and $L$ lends some insight into the dominant coupling physics in each of the regimes. Recall that $\mathbf{C}$ is a function of $a$ and $\Lambda$ is primarily a function of $L$. Thus, the amplitude $(a)$ of the wave will determine which modes are the dominant internal modes. The change in the relative amplitudes and phases of the dominant internal modes as they propagate through the ISW is primarily a function of $L$. In the analysis which follows, we will focus mostly on the change in relative phases of the modes because the differences between the attenuation of each of the acoustic modes is negligible while passing through an ISW.

\section{A. Cancellation Regime}

The cancellation regime consists of the region of small $L$ where significant modal coupling exists in the trough of the ISW but almost no coupling remains after the acoustic signal has passed through the entire ISW. Since $\mathbf{C}$ is a unitary matrix, we get $\mathbf{C}_{j}^{t} \mathbf{C}_{i}=\delta_{i j}$. Assume as before that modes $i, j, l, m$, etc., are the dominant internal modes. That is, $\mathbf{C}_{k i} \approx 0$ for $k \notin\{i, j, l, m, \ldots\}$. Then, as long as $L$ is small enough so that $\Lambda_{i i} \approx \Lambda_{j j} \approx \Lambda_{l l} \approx \Lambda_{m m} \ldots$., (24) will yield

$$
\mathbf{M}\left(L_{S}^{+}\right)_{j} \approx \Lambda_{i i} \mathbf{C}_{j}^{t} \mathbf{C}_{i} \mathbf{M}\left(-L_{S}^{-}\right)_{i}=\Lambda_{i i} \delta_{i j} \mathbf{M}\left(-L_{S}^{-}\right)_{i}
$$

Thus, very little net coupling will occur as the acoustic signal passes though the ISW. Physically, the significant acoustic modes do not propagate long enough within the trough of the ISW to change their relative phases and amplitudes. Interpreted another way, the ISW is so short that the vertical structure of the acoustic field does not change significantly as it passes though the ISW.

\section{B. Coupling Regime}

As the ISW horizontal scale $(L)$ is increased beyond the cancellation regime, the net coupling though the entire ISW increases. The region so characterized is the coupling regime. In the coupling regime, we expect the relative phases of the dominant internal modes as these modes propagate out of the trough of the ISW to be such that

$$
\left|\mathbf{C}_{i j} \Lambda_{i i} \mathbf{C}_{i i}+\mathbf{C}_{j j} \Lambda_{j j} \mathbf{C}_{j i}+\mathbf{C}_{l j} \Lambda_{l l} \mathbf{C}_{l i}+\mathbf{C}_{m j} \Lambda_{m m} \mathbf{C}_{m i}+\cdots\right|
$$

is large for some $j \neq i$. The larger the magnitude of this sum, the stronger the net coupling from mode $i$ to mode $j$. In addition, we expect

$$
\left|\mathbf{C}_{i i} \Lambda_{i i} \mathbf{C}_{i i}+\mathbf{C}_{j i} \Lambda_{j j} \mathbf{C}_{j i}+\mathbf{C}_{l i} \Lambda_{l l} \mathbf{C}_{l i}+\mathbf{C}_{m i} \Lambda_{m m} \mathbf{C}_{m i}+\cdots\right|
$$


to be relatively small in this region. Thus, for a fixed amplitude ISW, the values of $L$ at which the relative phases of the dominant internal modes are such that (26) is large and (27) is small will delineate the coupling regime. For an ISW with a fixed horizontal scale, the values of $a$ which yield dominant internal modes such that (26) is large and (27) is small will delineate the coupling regime.

\section{Transparent Resonance Pattern}

In some cases, the results generated with the PE show a range of $L$ values (for fixed $a$ ) out of the cancellation regime which exhibit significant internal coupling and little net coupling by the ISW. The ISW is apparently transparent for this mode. The SIA equations predict that as long as the ISW is not in the adiabatic regime (where the gradients are too weak to induce coupling) then transparent resonances should repeat periodically in $L$.

These regions are characterized by the values of $L$ for which the phases of the dominant internal modes differ by integer multiples of $2 \pi$. This can be expressed as

$$
\left(2 L k r_{l}^{(1)}-2 L k r_{m}^{(1)}\right) \approx 2 \pi n
$$

for some integer $n$. For all pairwise combinations of the dominant internal modes (28) can be rewritten as

$$
L \approx \frac{\pi n}{k r_{l}^{(1)}-k r_{m}^{(1)}}=n X_{l, m} / 2
$$

where $X_{l, m}$ is the mode interference distance discussed earlier. We refer to these regions as transparent resonances.

Given the phase realignment of the dominant internal modes at these transparent resonances, the unitary property of $\mathbf{C}$ means that

$$
\mathbf{M}\left(L_{S}^{+}\right)_{j} \approx \Lambda_{i i} \mathbf{C}_{j}^{t} \mathbf{C}_{i} \mathbf{M}\left(-L_{S}^{-}\right)_{i}=\Lambda_{i i} \delta_{i j} \mathbf{M}\left(-L_{S}^{-}\right)_{i}
$$

Note that (25) and (30) are the same equation. For the cases tested, at most one transparent resonance was found using the PE before the ISW entered the adiabatic regime at high $L$.

\section{Adiabatic Regime}

In the adiabatic regime, the horizontal gradients in the sound-speed profile are insufficient to induce coupling between the acoustic modes. In this regime, the SIA is not effective as a method of describing the relevant physics governing the propagation of the modes. However, both the analytic adiabatic invariance calculations and the PE modeling allow determination of ISW effects in this regime, and the two methods have been shown to agree.

\section{E. Sensitivity to ISW Amplitude}

The amplitudes of the ISW affect the mode coupling in two ways. The first and most obvious is that for a fixed $L$, the larger values of $a$ result in larger horizontal gradients of the sound speed. Generally, these larger gradients result in more mode coupling.

A second effect of the amplitude is the determination of which acoustic modes are the dominant internal modes. Note that the adiabatic invariance formulas provide $\beta_{i j}(a, x / L)$ for given $L$ and $a$, with larger $\beta_{i j}(a, x / L)$ values indicating more coupling between the $i$ th and $j$ th modes. However, $\beta_{i j}(a, x / L)$ depends upon $U_{i j}(x / L)$ which is a weighted inner product of the mode shapes over the transition layer of the water column. Since different values of $a$ have different transition layer locations, the inner product varies, as does the coupling between modes. In the context of the SIA representation, the relationship between soliton amplitude and the dominant internal modes is reflected in the coupling matrix $\mathbf{C}$. The elements of $\mathbf{C}$ are simply the projections of the respective mode shapes inside and out of the wave troughs.

The dominant internal modes effect the net coupling in an ISW because the dominant internal modes determine at which horizontal scales $(L)$ the coupling will be characterized by cancellation, coupling, or transparent resonance. The sensitivity of the overall coupling to $a$ appears to be less than the sensitivity to $L$.

\section{3-D PROPAGATION THROUGH ISWs}

The 2-D PE and 2-D SIA models have assumed colinear acoustic and ISW horizontal wavevectors, with the soundspeed gradient orthogonal to these equal to zero. In this case, neither the acoustic field nor the modes experience horizontal refraction [18], [19], and coupling can be modeled with 2-D propagation. However, when the acoustic and ISW horizontal wavevectors are not colinear, the acoustic signal encounters a lateral gradient in the sound speed, and the signal will refract horizontally. This effect must be either accounted for or discounted when applying the 2-D mode coupling results. It is shown here that refraction can be discounted for angles up to $65^{\circ}$. An acoustic signal composed of a single vertical acoustic mode with a horizontally planar wavefront is examined here. The more stringent case of nonplanar wavefronts is left for future analysis.

Our premise is that 2-D modeling is sufficient becuase modes do not significantly refract, and phase relationships in the ISW trough which control coupling are not disturbed. With this premise, the application of 2-D modeling to the 3-D noncolinear case is handled simply by scaling the ISW

$$
L_{\mathrm{eff}}=L / \cos \theta \text {. }
$$

Here, $L_{\text {eff }}$ is the effective horizontal scale of the ISW, $L$ is the physical scale of the ISW, and $\theta$ is the horizontal angle between the acoustic and ISW wavevectors (the angle of incidence). Thus, the 2-D approach models the 3-D case by stretching the ISW. The validity of this approach is evaluated here.

Fig. 16 shows the horizontal ray paths of acoustic modes 1 and 3 as they pass through an example ISW with an initial angle of incidence of $60^{\circ}$. The coupling effects of the soliton are ignored, and the plots show the paths in the case of adiabatic mode propagation. We refer to this as intramode refraction. The path for the $i$ th mode is computed using the ray equations [13], [18]

$$
d \mathbf{r} / d s=\mathbf{k r}_{i} / k r_{i}, \quad d \mathbf{k r}_{i} / d s=\nabla k r_{i} .
$$

Here, $k r_{i}$ denotes the horizontal wavenumber of the $i$ th mode, and $\theta_{i}$ is the direction of $\mathrm{kr}_{i}$. The paths were computed numerically. Fig. 16 clearly shows the different refraction of the two modes and the difference between their paths. 


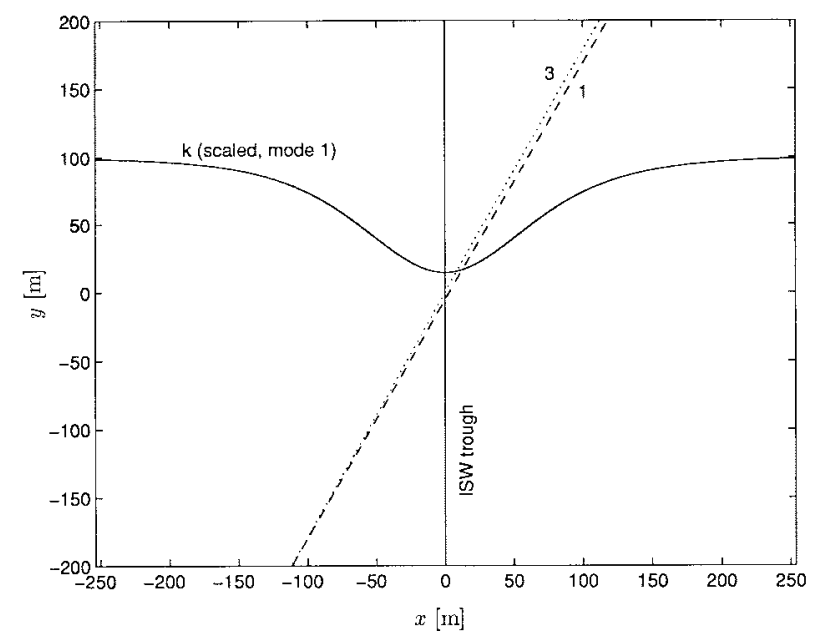

Fig. 16. The horizontal paths of acoustic modes 1 (dashed line) and 3 (dotted line) are shown crossing an ISW of $a=15, L=100 \mathrm{~m}$ at $x=0$. The angle between the acoustic and ISW propagation is $60^{\circ}$ at $x$ far from zero. The profile of mode 1 wavenumber $k_{1}$ is shown in scaled form. The maximum of $k_{1}$ is 1.6803 and the minimum is 1.6775 .

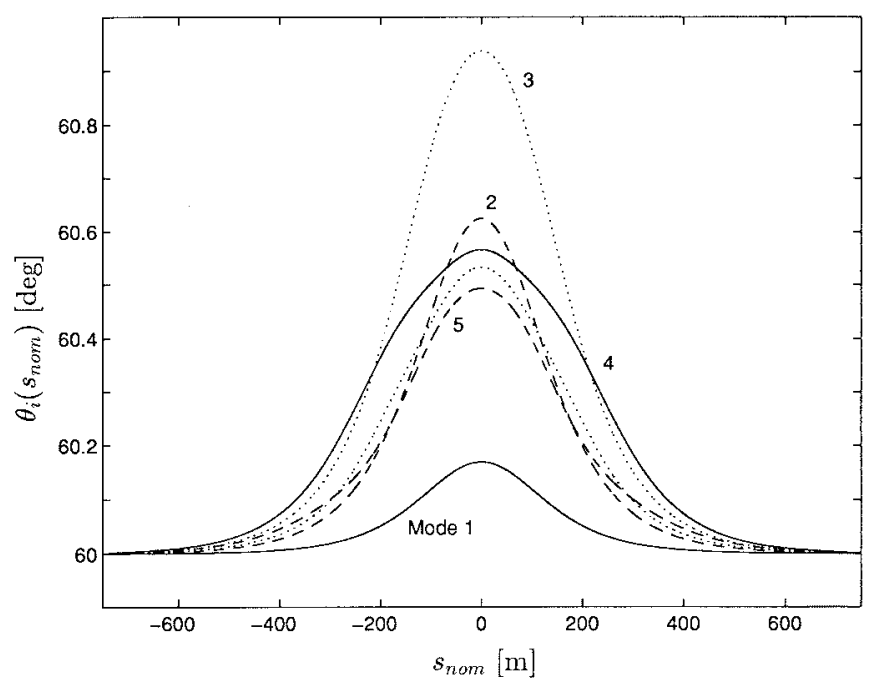

Fig. 17. Angle of propagation relative to ISW direction is plotted for acoustic modes $1-6$. The ISW has $a=15$ and $L=100$. The angle distant from the ISW is $60^{\circ}$, as in Fig. 16. The independent variable is nominal path $s$ as it would exist with no soliton. The modes are indicated by line type, as in Fig. 9, for example.

Adiabatic propagation angles for modes 1-6 are shown in Fig. 17. Angle is plotted as a function of distance along the nominal (unrefracted) mode path $\left(s_{\text {nom }}\right)$, and an initial horizontal angle of incidence is $60^{\circ}$. Note that the angular spread between the directions of each of the modes is always less than $1.0^{\circ}$. Each mode returns to its original direction after passing through the ISW.

In addition to the intramode refraction described above, intermode refraction will occur as energy is coupled from a mode with a particular horizontal wavenumber (phase velocity) to another mode with a different horizontal wavenumber. This intermode refraction will occur continually as mode coupling occurs within the body of the ISW. However, without accounting for the continual coupling and refraction, the SIA can be used to perform a first-order analysis of the effect of the intermode and intramode refraction on the net coupling

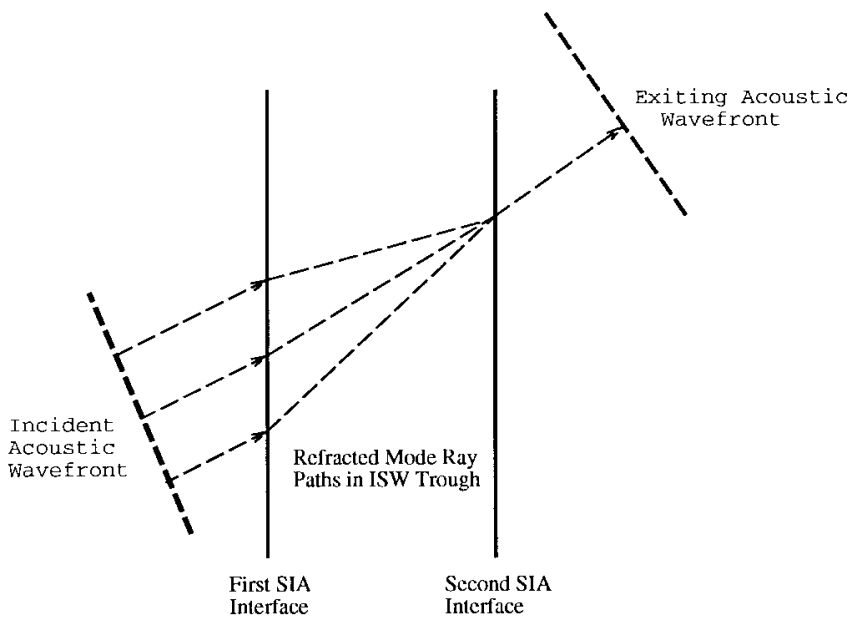

Fig. 18. The offset along the initial acoustic wavefront of modes that exit the ISW in unison, after refraction, are shown schematically for the SIA geometry.

induced by the ISW.

In utilizing the SIA, we use the following form of Snell's Law:

$$
k r_{i}^{(0)} \sin \theta_{i}^{(0)}=k r_{j}^{(1)} \sin \theta_{j}^{(1)} .
$$

The superscripts (1) and (0) indicate inside and outside the ISW trough, respectively, and the subscript indicates mode index. Assume that the incident acoustic signal is composed of a single vertical acoustic mode (the $i$ th mode) with a horizontally planar wavefront. Further, assume that the incident energy in the $i$ th mode couples to the $l$ th mode upon entering the trough of the ISW and this energy couples into the $j$ th mode as it exits the trough of the ISW. Then

$$
k r_{i}^{(0)} \sin \theta_{i}^{(0)}=k r_{l}^{(1)} \sin \theta_{l}^{(1)}=k r_{j}^{(0)} \sin \theta_{j}^{(0)} .
$$

Note that given $i, j, \theta_{i}^{(0)}, k r_{i}^{(0)}$, and $k r_{j}^{(0)}, \theta_{j}^{(0)}$ is independent of $\theta_{l}^{(1)}, k r_{l}^{(1)}$, and the index $l$. Thus, the direction of mode $j$ upon exiting the ISW is independent of the index and horizontal wavenumber of the mode in which the energy propagated while in the trough of the ISW. This independence does not hold for modal phase, however.

The effect of the index and horizontal wavenumber of the mode in which the energy propagated while in the trough of the ISW is seen in the horizontal offset of the modal ray paths. The signal from the $i$ th incident mode which couples into different modes upon entering the ISW will follow different ray paths within the trough as determined by (34). This will result in different horizontal offsets of the modal ray paths within the ISW and upon exiting the ISW, as illustrated in Fig. 16. The result of this path offset is that the signal in the $i$ th incident mode from different entrance points to the ISW will coherently couple into the $j$ th mode at a single exit point from the ISW and will propagate along a single path after exiting the ISW. This is illustrated in Fig. 18. The horizontal offset of the appropriate entrance points to the ISW is determined by the refraction of the ray paths of each of the modes within the trough of the ISW.

Recall from 2-D SIA analysis that the relative phasing of mode energy upon exiting the ISW is the primary determinant of the net coupling. Differences between modal ray path 


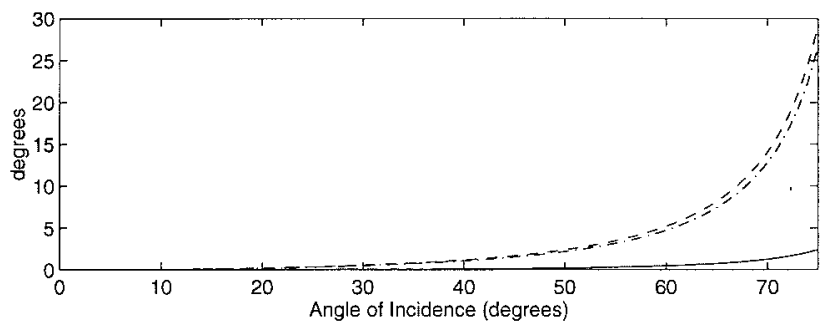

(a)

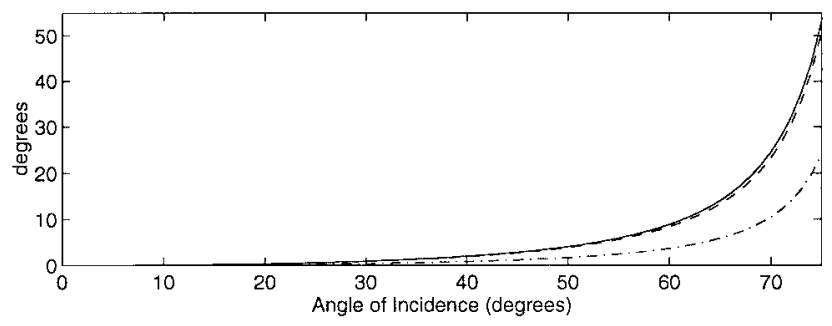

(b)

Fig. 19. Phase differences between the "stretched 2-D" modes moving through ISW's and 3-D refracted and coupled modes are plotted versus angle of incidence. (a) Three examples are shown, all involving relative phasing between modes $1-3$. The cases are $\delta 3_{12}^{2}-\delta 2_{12}$ (line), $\delta 3_{13}^{2}-\delta 2_{13}$ (dash), and $\delta 3_{23}^{2}-\delta 2_{23}$ (dash-dot). (b) Three examples are shown involving relative phasing between mode 4 and modes $1-3$. The three cases are $\delta 3_{14}^{2}-\delta 2_{14}$ (line), $\delta 3_{24}^{2}-\delta 2_{24}$ (dash), and $\delta 3_{34}^{2}-\delta 2_{34}$ (dash-dot).

lengths within the ISW can therefore effect the net coupling. The appropriate path to consider is the refracted ray path from each of the horizontally offset points on the incident acoustic wavefront to a single exit point. Assuming that the energy in the incident acoustic wavefront is contained in the $i$ th mode, the relative phasings of the refracted signal in each of the modes at the exit point from the trough of the ISW are given by

$$
\lambda_{l}^{i}=d_{i l}^{(0)} k r_{i}^{(0)}+d_{i l}^{(1)} k r_{l}^{(1)} .
$$

$d_{i l}^{(0)}$ is the horizontal path length from the appropriate point on the incident acoustic wavefront to the entrance point to the trough of the ISW for the path for the lth refracted mode. $d_{i l}^{(1)}$ is the length of the refracted path for the $l$ th mode in the trough of the ISW. To determine the validity of the use of the "stretched" 2-D approach to modeling 3-D propagation, we first compare the modal phase differences calculated using (35):

$$
\delta 3_{l m}^{i}=\lambda_{l}^{i}-\lambda_{m}^{i}
$$

with those calculated using the "stretched" 2-d modeling

$$
\delta 2_{l m}=2 L_{\mathrm{eff}} k r_{l}^{(1)}-2 L_{\mathrm{eff}} k r_{m}^{(1)} \text {. }
$$

If the differences between $\delta 3_{l m}^{i}$ and $\delta 2_{l m}$ are small for all of the modes into which the $i$ th mode couples significant energy, then the "stretched" 2-D modeling will reasonably predict the net coupling which would be introduced by the ISW in the 3 -D case. Fig. 19 shows $\delta 3_{l m}^{i}-\delta 2_{l m}$ for the case where $a=12, L_{\text {eff }}=100 \mathrm{~m}$, and $i=2$. The plots shows reasonable phase agreement between the "stretched" 2$\mathrm{D}$ and 3-D modeling approaches up through about $60^{\circ}$ angle of incidence and rapidly degrading agreement after a $70^{\circ}$ angle.
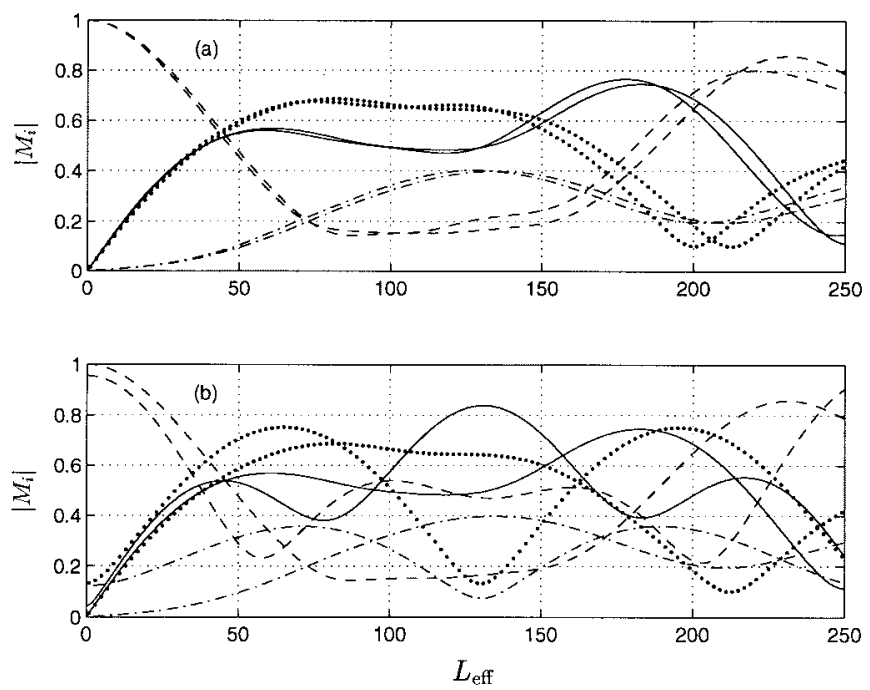

Fig. 20. The normalized SIA-computed coupled-mode amplitudes are compared for the 3-D and the "stretched 2-D" models. Incident energy is in mode 2. Amplitude at exit is shown for mode 1 (line), mode 2 (dash), mode 3 (dots) and mode 4 (dash-dot). The 2-D curve is identical in the two frames, thus it can be identified. The 3-D curve varies between the frames: (a) angle of incidence $65^{\circ}$ and (b) angle of incidence $80^{\circ}$.

The calculation of net modal coupling using the SIA approximation confirms this conclusion. Fig. 20 shows these results. As before, we use an ISW amplitude of $12 \mathrm{~m}$ with the energy in the incident acoustic wavefield contained in mode 2 . The normalized modal amplitudes of the acoustic wavefield after exiting the ISW are calculated using (21) and plotted as a function of $L_{\text {eff }}$. For the "stretched" 2-D model, $L_{\text {eff }}$ is substituted for $L_{S}$ in (21). For the SIA 3-D model, $\lambda_{l}^{2}$ as calculated in (35) is substituted for $k r_{l}^{(1)} 2 L_{S}$ in (18). The results show very close agreement between the mode amplitudes calculated using the two different models for the case when the angle of incidence is $65^{\circ}$. For the case when the angle of incidence is $80^{\circ}$, the agreement is much worse.

The above results indicate that for angles of incidence up to approximately $65^{\circ}$, the net coupling induced by the ISW can be reasonably modeled by substituting $L_{\text {eff }}$ for $L_{S}$ in (18). This result can be expected to be valid over the range of effective horizontal scales $\left(L_{\mathrm{eff}}\right)$ over which the SIA is itself valid. For effective horizontal scales greater than this range, either significant coupling will occur which cannot be modeled in this manner or propagation through the ISW will be adiabatic and no coupling would occur. For the former case, more analysis is needed to determine the nature of the ISW induced modal coupling. As stated earlier, this analysis has assumed a horizontally planar incident acoustic wavefront. Further analysis is needed for the nonplanar wavefront case.

\section{SUMMARY}

The coupling behavior of acoustic modes propagating though continental-shelf internal solitary waves has been examined. Using a sharp interface approximation, the relative phases of modes propagating through the troughs of ISW's is shown to be the dominant factor in determining the amount of net mode coupling induced by the ISW. For the case studied in detail, $400 \mathrm{~Hz}$ and a three-layer system, this generally holds 
for horizontal ISW scales of less than $200 \mathrm{~m}$.

The relative phase changes in the modes can be used to predict the existence of four physical regimes. The cancellation regime has no net coupling occurring at intermediate scales but occurs for waves with small horizontal scale. Regimes of net coupling appear for longer waves, as do periodically occurring regimes of little net coupling, referred to as transparent resonances. The fourth regime is adiabatic propagation, the long-ISW limiting case, which occurs for scales above a limit which varies with mode number, stratification, etc. The cases of cancellation and transparent resonance exhibit strong coupling within the ISW, with little or no net coupling. In the cases examined, the onset of adiabaticity at increasing horizontal ISW scale prevents the numerical reproduction of more than one region of transparent resonance at moderate $L$, with the would-be resonant situation at higher $L$ absent because modes do not couple.

An analysis of horizontal mode refraction within ISW's for acoustic paths oblique to the ISW troughs indicates that the differences between the directions and the lengths of the modal paths vary negligibly with incidence angle. Therefore, with an accordant stretching of the effective horizontal scale of the ISW by a factor of $\cos ^{-1} \theta, 2$-D propagation models can be used to predict behavior for a wide range of incidence angles. Although not shown here, our computations show that the effects of loss in the bottom are a secondary influence on the evolution of acoustic mode coefficients within ISW's in comparison to those highlighted here.

\section{ACKNOWLEDGMENT}

The FEPE program was developed and distributed by $\mathrm{M}$. Collins of NORDA and of the Naval Research Laboratory. The authors thank A. Newhall and C. Sellers for managing the computing resources.

\section{REFERENCES}

[1] J. M. Huthnance, "Internal tides and waves near the continental shelf edge," Geophys. Astrophys. Fluid Dynamics, vol. 48, pp. 81-106, 1989

[2] L. A. Ostrovsky and Y. A. Stepanyants, "Do internal solitons exist in the ocean?," Rev. Geophys., vol. 27, pp. 293-310, 1989.

[3] J. R. Apel, L. A. Ostrovsky, and Y. A. Stepanyants, "Internal solitons in the ocean," The Johns Hopkins Univ. Applied Physics Lab., Laurel, MD, Rep. MERCJRA0695, 1995.

[4] D. M. Milder, "Ray and wave invariants for SOFAR channel propagation," J. Acoust. Soc. Amer., vol. 46, pp. 1259-1263, 1969.

[5] J. Zhou, X. Zhang, and P. H. Rogers, "Resonant interaction of sound wave with internal solitons in the coastal zone," J. Acoust. Soc. Amer., vol. 90, pp. 2042-2054, 1991.

[6] A. K. Liu, "Analysis of nonlinear internal waves in the New York Bight," J. Geophys. Res., vol. 93, pp. 12317-12329, 1988.

[7] J. R. Apel, M. Badiey, C.-S. Chiu, S. Finette, R. Headrick, J. Kemp, J. Lynch, A. Newhall, M. Orr, B. Pasewark, D. Tielbuerger, A. Turgut, K. von der Heydt, S. Wolf, and The SWARM Group, "An overview of the 1995 SWARM shallow water internal wave acoustic scattering experiment," IEEE J. Oceanic Eng., to be published.

[8] H. Sandstrom and N. S. Oakey, "Dissipation in internal tides and solitary waves," J. Phys. Oceanogr., vol. 25, pp. 604-614, 1995.

[9] L. I. Schiff, Quantum Mechanics. New York: McGraw-Hill, 1968.
[10] J. Lighthill, Waves in Fluids. New York: Cambridge Univ. Press, 1978

[11] G. B. Witham, Linear and Nonlinear Waves. New York: Wiley, 1974

[12] D. Bogucki and C. Garrett, "A simple model for the shear-induced decay of an internal solitary wave," J. Phys. Oceanogr., vol. 23, pp. 1767-1776, 1993.

[13] A. D. Pierce, "Extension of the method of normal modes to sound propagation in an almost stratified medium," J. Acoust. Soc. Amer., vol. 37, pp. 19-27, 1965

[14] M. D. Collins, "FEPE user's guide," Naval Ocean Research and Development Activity, Stennis Space Center, MS, NORDA Tech. Note 365, 1988.

[15] L. B. Dozier and F. D. Tappert, "Statistics of normal mode amplitudes in a random ocean. I. Theory," J. Acoust. Soc. Amer., vol. 63, pp. 353-365, 1978.

[16] M. D. Collins and E. K. Westwood, "A higher-order energy-conserving parabolic equation for range-dependent ocean depth, sound speed, and density," J. Acoust. Soc. Amer., vol. 89, pp. 1068-1075, 1991.

[17] F. B. Jensen, W. A. Kuperman, M. B. Porter, and H. Schmidt, Computational Ocean Acoustics. New York: Amer. Inst. Phys., 1994.

[18] K. D. Heaney, W. A. Kuperman, and B. E. McDonald, "Perth-Bermuda sound propagation (1960): Adiabatic mode interpretation," J. Acoust. Soc. Amer., vol. 90, pp. 2586-2594, 1991.

[19] H. Weinberg and R. Burridge, "Horizontal ray theory for ocean acoustics," J. Acoust. Soc. Amer., vol. 55, pp. 63-79, 1974.

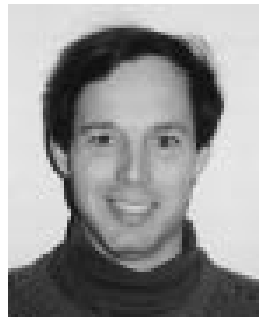

James C. Preisig (S'87-M'91) received the B.S degree in electrical engineering from the United States Coast Guard Academy in 1980, the S.M. and E.E. degrees in electrical engineering from the Massachusetts Institute of Technology, Cambridge, in 1988, and the Ph.D. degree in electrical and ocean engineering from the Massachusetts Institute of Technology/Woods Hole Oceanographic Institution Joint Program in Oceanography and Oceanographic Engineering, Woods Hole, MA, in 1992.

Since 1994, he has been a Visiting Assistant Professor in the Department of Electrical and Computer Engineering at Northeastern University, Boston, MA, and a Visiting Investigator at the Woods Hole Oceanographic Institution. His research interests are in the areas of adaptive signal processing, signal propagation modeling, and numerical optimization. His particular research programs cover these three interrelated areas. The first is the development of a better understanding of the effect which environmental fluctuations have on propagating acoustic and electromagnetic signals. The second is to use this understanding to develop adaptive signal processing algorithms with improved performance characteristics. The final area is the development of computationally robust and numerically efficient techniques for implementing new adaptive algorithms.

Dr. Preisig is a member of the Underwater Acoustic Signal Processing Technical Committee.

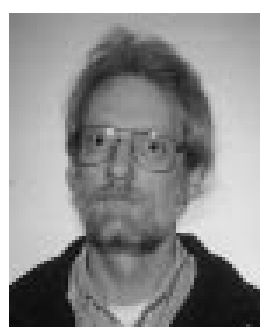

Timothy F. Duda received the B.A. degree in physics from Pomona College, Claremont, CA, in 1979 and the Ph.D. degree in oceanography from Scripps Institution of Oceanography, University of California, San Diego, in 1986.

He has been a Scientist at the Woods Hole Oceanographic Institution since 1991, and prior to that was a Research Scientist at the University of California, Santa Cruz. His two primary fields of study are the measurement and characterization of ocean internal waves and microstructure, and ocean acoustic propagation. He has modeled and analyzed fluctuations of ocean acoustic transmissions, has made electromagnetic-type internal-wave measurements, and performed in situ measurement of ocean microstructure.

Dr. Duda is a member of the American Meteorological Society, the American Geophysical Union, and the Acoustical Society of America. 